\title{
Generation of amorphous carbon models using liquid quench method: A reactive molecular dynamics study
}

\author{
Raghavan Ranganathan ${ }^{1}$, Srujan Rokkam ${ }^{2 \dagger}$, Tapan Desai ${ }^{2,3}$ and Pawel Keblinski ${ }^{1}$ \\ ${ }^{1}$ Department of Materials Science and Engineering, Rensselaer Polytechnic Institute, Troy, New \\ York, 12180, USA \\ ${ }^{2}$ Advanced Cooling Technologies, Inc., 1046 New Holland Ave., Lancaster, Pennsylvania, \\ 17601, USA \\ ${ }^{3}$ Current affiliation: JLC Electromet Pvt Ltd.,VKIA, Jaipur, Rajasthan, 302013, India
}

\begin{abstract}
Using molecular dynamics simulations with a reactive force field (ReaxFF), we generate models of amorphous carbon $(a-C)$ at a wide range of densities (from $0.5 \mathrm{~g} / \mathrm{cc}$ to $3.2 \mathrm{~g} / \mathrm{cc}$ ) via the 'liquid-quench' method. A systematic study is undertaken to characterize the structural features of the resulting $a-C$ models as a function of carbon density and liquid quench simulation conditions: quench rate, type of quench (linear or exponential), annealing time and size of simulation box. The structural features of the models are investigated in terms of pair correlation functions, bond-angles, pore-size distribution and carbon hybridization content. Further, the influence of quench conditions on hybridization/graphitization is investigated for different stages of the simulation. We observe that the structural features of generated a-carbon models agree well with similar models reported in literature. We find that in the low-density regime, size
\end{abstract}

\footnotetext{
${ }^{+}$Corresponding author. E-mail: srujan.rokkam@1-act.com (Srujan Rokkam), Phone: (717) 2956059
} 
effects play an important role in determining the pore size distribution and the structures are predominantly anisotropic. Whereas, at densities larger than $1.0 \mathrm{~g} / \mathrm{cc}$, the structures are spacefilling and differences exist only in terms of carbon hybridization. The rate of structural evolution (pore size and hybridization) during the quench process is observed to be dependent on the quench type, rate and the annealing time.

Keywords: Amorphous carbon; liquid quench; quench MD; ReaxFF; structural characteristics; char

\section{Introduction}

Carbon shows remarkable versatility since it exists in various chemical and structural forms. On one hand, crystalline and ordered phases such as graphene, diamond, carbon nanotubes, etc., confer an extraordinary range of properties. On the other, equally important is the plethora of amorphous structures of carbon (denoted as $a-C$ and alternately referred to as disordered carbon) existing in a wide range of densities ranging from low-density, char-like carbon to high-density diamond-like and tetrahedral amorphous carbon (denoted as $t a-C$ [1]), with varied structural and chemical features. Correspondingly, this confers $a-C$ with a wide variety of properties and applications, ranging from low-conductivity heat-shield ablators $[2,3]$ in the low-density regime, to high-hardness, chemically inert and optically transparent coatings [4,5], magnetic storage applications [6] among others [5] for diamond-like amorphous carbon films.

The term "amorphous carbon" can be attributed as an umbrella term to carbon at a large range of densities ranging from char-like carbon $(\sim 0.2$ to $0.5 \mathrm{~g} / \mathrm{cc}[3])$ to high-density, diamond- 
like carbon $(\sim 3.2 \mathrm{~g} / \mathrm{cc}[5])$. Since there is no well-defined order for amorphous carbon, it has been a challenge to characterize and fully understand their structure [7]. It is in this regard that models of $a-C$ structure generated by computer simulation techniques become very useful in understanding complex structure-property relations and optimize desired properties.

A large body of work exists on modeling $a-C$ at various densities and conditions ranging from thin films [8,9] to bulk [10-14]. They can broadly be classified broadly into three simulation methodologies: (a) assembling structural units of amorphous carbon elements guided by general experimental observations; (b) Reverse Monte Carlo (RMC) based methods, and (c) mimetic approach involving quench molecular dynamics simulations (also referred to as "liquidquench"). Among the first class of models, the earliest model is the simplistic split-pore model [15-18] consisting of graphite blocks separated by a pore. Biggs et. al. [19] improved the split pore model by constructing 'virtual porous carbon (VPC)' that had several structural building blocks (usually crystallites of graphene layers) consisting of topological features to mimic pore sizes and structural features as observed in experiments. Beeman et. al. [10] constructed arbitrary models of amorphous carbon at four densities from $2.1 \mathrm{~g} / \mathrm{cc}$ to $3.4 \mathrm{~g} / \mathrm{cc}$ with varying hybridizations and ring sizes. By comparing the computed vibrational spectra of the models to experimental Raman spectra, they deduced that a-C at these densities consists of predominantly (close to 90\%) $s p^{2}$ hybridized carbon. Harris et. al. [12] modeled microporous carbon built from fullerene-like fragments that consist of five, six and seven-membered rings, led by experimental observation of such units using Transmission electron microscopy. Powles et. al. [20] used a similar strategy to construct ordered $s p^{2}$ hybridized model networks of carbon consisting of various precursors of disordered carbon. 
The second class of models are constructed based on Reverse Monte Carlo (RMC) method [13,14,21-27], a reconstruction technique, whereby, some structural information usually in the form of pair correlation functions, or alternatively, the structure factor as obtained from diffraction data (X-ray or neutron diffraction), is set as a target and a Monte Carlo algorithm evolves the model to new configurations that minimize the difference between the simulated structural feature and the target. Variations of the technique include adding constraints (Constrained Reverse Monte Carlo method [25]) in the form of average bond angles and coordination numbers and, the addition of a potential energy term for the structure obtained from a reactive force field (denoted as Hybrid Reverse Monte Carlo method [23]).

The third class of methodology for $a-C$ models constitute mimetic methods that try to capture the chemistry of formation of disordered carbon. The most popular of this class of methods is the liquid quench route, which forms the basis of this work. In this method, carbon is quenched from a high-temperature "liquid" state to a low temperature solid. Starting with the efforts to model $a$ - $C$ using first-principles based approaches [28,29], the technique has come a long way to using highly descriptive force-fields to capture the reaction chemistry. For example, Marks developed the Environment Dependent Interaction Potential (EDIP) for carbon and has used it to model $a-C[30,31]$ and showed better prediction of properties compared to the traditional reactive potentials like Tersoff and REBO. Saurez-Martinez and co-workers [32] used this potential to study disordered carbon at relatively high densities $(1.4 \mathrm{~g} / \mathrm{cc}$ to $3 \mathrm{~g} / \mathrm{cc})$ to study structure-thermal property relationship. Shi developed the computationally efficient Reaction Summation State (RSS) potential [33] that describes $s p^{2}$ hybridized carbon and employed it to satisfactorily model $a-C$ at densities between $0.75 \mathrm{~g} / \mathrm{cc}$ to $1.5 \mathrm{~g} / \mathrm{cc}$. The same potential was used by Palmer et. al [34] to model "carbide-derived carbon", an amorphous form of carbon at density 
close to $0.9 \mathrm{~g} / \mathrm{cc}$. A somewhat allied technique has been used by Makeev et. al $[35,36]$ where bulk polyethylene (as described by the REBO reactive potential) was pyrolized, and the resulting volatile gases were removed to obtain a porous, amorphous carbon (char). We refer the reader to the excellent review of $a-C$ materials by Marks [7] for a more detailed analysis on the merits and shortcomings of various modeling approaches.

In this work, we use the liquid-quench method and the reactive force-field, ReaxFF [37] to generate models of $a-C$ at various densities ranging from $0.5 \mathrm{~g} / \mathrm{cc}$ to $3.2 \mathrm{~g} / \mathrm{cc}$. We have previously used this method to generate $a-C$ at $0.5 \mathrm{~g} / \mathrm{cc}$ and $1.0 \mathrm{~g} / \mathrm{cc}$ (corresponding to mesoporous and microporous carbons respectively) and characterized high-temperature diffusion of gas molecules in these complex structures [38,39]. The advantage of using ReaxFF is that there is no need for experimental inputs like for the Reverse Monte Carlo method, and the relatively high accuracy in the description of reactivity makes it ideal for capturing the carbon chemistry accurately. Recently, Li et. al. [40] compared the use of ReaxFF with the REBO and Tersoff potentials to model $a-C$ at densities between $2 \mathrm{~g} / \mathrm{cc}$ and $3.2 \mathrm{~g} / \mathrm{cc}$ using the liquid quench method and found that ReaxFF is better at describing features such as hybridization and $C$-C bond lengths at wider density ranges, though the less computationally expensive potentials (Tersoff and $R E B O$ ) could be used to model specific density regimes.

In our work, we observe that the structural features of $a-C$ across the entire density spectrum (from $0.5 \mathrm{~g} / \mathrm{cc}$ to $3.2 \mathrm{~g} / \mathrm{cc}$ ) agree well with other models reported in literature. The effects of various parameters in the liquid-quench method, namely, the quenching rate, the annealing time, and simulation cell size are investigated in detail. Further, two different types of quenching methods (exponential and linear) which control the rate of system temperature drop are investigated. Also of importance is the size effects in generating $a-C$ at lower densities (less 
than $\sim 1.0 \mathrm{~g} / \mathrm{cc}$ ), where we show that increasing simulation cell sizes leads to larger pore sizes, while the same carbon hybridization is maintained.

The paper is organized as follows. In section 2, the simulation methodology for generation of these structures and the various structural characterization methods are explained. Section 3 contains the results and discussion on the effect of various simulation parameters on the final $a-C$ structure and the conclusions are presented in section 4 .

\section{Simulation overview and tools}

\section{1 Liquid quench method}

The model carbon structures used in this study were generated using the liquid-quench method [28,41] and a reactive force field, ReaxFF [37]. The liquid quench method lends itself well to model amorphous structures, especially amorphous carbon [32,42,43], gels [44] and inorganic oxides [41]. ReaxFF is a reactive potential, which uses the concept of bond order to model the chemical interactions within a reactive system. The bond order based definition of atomic and molecular interactions excludes the need for predefined reactive sites (or reaction pathways) and allows for description of dynamic bond breaking and formation. The ReaxFF force field parameters are derived solely from Quantum Mechanics and provide an unbiased representation of the reaction chemistry. Thus ReaxFF potentials can be directly applied to novel systems that may not have been studied experimentally. Using the liquid quench method in conjunction with ReaxFF allows us to effectively model various $a$ - $C$ structures across a wide variety of densities, encompassing char to glassy carbon to diamond-like carbon. We note that among various reactive potentials for carbon (Tersoff [45], REBO [46,47], EDIP [30] and RSS [33]), ReaxFF is the most comprehensive one which captures the chemistry most accurately. 
The steps involved in the overall process are - (a) Start with $N$ carbon atoms placed at random positions in a simulation cell with fully periodic boundaries, ramp the temperature to a

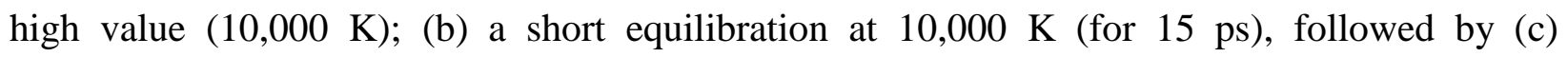
quenching the system to $3,000 \mathrm{~K}$ (via rescaling of atomic velocities at each step) with a quench rate $Q$. Following this, the system was (d) annealed at $3,000 \mathrm{~K}$ for time $t_{a}$ and finally (e) quenched and equilibrated at $300 \mathrm{~K}$ for about $10 \mathrm{ps}$. The overall procedure is outlined as a flowchart in Figure 1. It should be noted that the choice of the temperature ranges in our liquid quench simulations does not have a physical significance, and are similar to values used in literature [32-34]. At the high temperature equilibration $(10,000 \mathrm{~K})$, the model mimics a gas phase and the following quench and anneal stages mimic the formation of disordered carbon. We use the ReaxFF force field for hydrocarbons that was developed by Chenoweth et. al. [48] and has been used successfully to model reactions in various forms of carbon like graphene [49] and carbon nanotubes [50]. All the ReaxFF parameters used in our studies correspond to ref. [47] with the ' $\mathrm{C} 2$ ' parameter set to 70.0 to correct for the formation of unphysical carbon-carbon units following the work of Nielson et. al [51] and the parameter set provided with the LAMMPS distribution.

A small time step of $0.07 \mathrm{fs}$ was chosen to ensure proper energy conservation at the high temperatures employed in this work. For the constant temperature annealing stage, a Berendsen thermostat with a damping constant of $100 \mathrm{fs}$ was used. The volume was fixed during the entire simulation process. All simulations were performed using the LAMMPS simulation package [52] using the version of ReaxFF implemented in C, namely, the "USER-REAXC" package.

\subsection{Simulation variables}


Using the liquid quench method, $a-C$ at various densities were generated, ranging from $0.5 \mathrm{~g} / \mathrm{cc}$ (mesoporous carbon mimicking char) to $3.2 \mathrm{~g} / \mathrm{cc}$ (diamond-like carbon). Naturally, a non-equilibrium process like liquid quenching has a large set of variables that could potentially affect the structural features. Most important of these are the quench rate, the annealing time and the system size simulated. In this work, we study the effect of all the above modeling variables on the final structure. For the quench step, studies reported in literature have used either linear (e.g. $[28,53])$ or exponential (e.g. $[31,40])$ quench steps. So, we also explore the effect of both linear and exponential quench routes for generating $a-C$ in this work. We find that the simulation cell size plays a crucial role on the resultant pore sizes, for the lower density structures $(0.5 \mathrm{~g} / \mathrm{cc}$ and $1.0 \mathrm{~g} / \mathrm{cc}$ ), with larger simulation cell leading to larger pore sizes.

We also note that since the liquid quench method involves a random placement of atoms at the initial step, this can potentially lead to structures having widely different pore features, for the same density. We have previously showed that this effect is prominent for the case of mesoporous (with a density $0.5 \mathrm{~g} / \mathrm{cc}$ ) and microporous (with a density $1.0 \mathrm{~g} / \mathrm{cc}$ ) amorphous carbon, where anisotropic structural features could arise, which have a direct bearing on structural features and properties such as gas diffusion rates and thermo-mechanical properties [38]. For those lower density regimes, we also showed that multiple realizations of structures were required at each density to obtain statistically meaningful properties [38]. Thus, in this work, for each simulation variable, multiple realizations of structures (three to five) were generated and structural features (such as pair correlation function, bond-angle distribution, ring size distribution, pore size, and carbon hybridization) were averaged over these samples.

Since ReaxFF is computationally expensive (requires an order of magnitude lower time step and runs about 1 to 2 orders of magnitude slower than conventional reactive and non- 
reactive potentials [54]), we are limited by the total simulation times (in terms of quench rates and annealing time) for reasonably large structures. The current simulation time-spans yield reasonable models of amorphous carbon for the entire range of densities as described in section 3.

\subsection{Structural characterization of generated amorphous carbon}

A detailed structural characterization of the generated structures has been carried out, including the pair correlation function, $g(r)$, angles distribution function, ring size distribution, pore size distribution (for $0.5 \mathrm{~g} / \mathrm{cc}$ and $1.0 \mathrm{~g} / \mathrm{cc}$ structures) and carbon hybridization. The pair correlation, angles and ring distribution were calculated using the Isaacs software [55]. For ring sizes, we use Guttmann's definition [56] according to which, a ring is denoted as the shortest path between two nearest neighbors. To characterize the pore sizes of these model structures, we find the pore size distribution (PSD) using the tool Zeo++ [57], which uses Voronoi decomposition for describing the pore geometry. Using a probe molecule (of radius $1.2 \AA$ in the current study), the code finds the change in accessible volume with respect to the probe size from, which relates to the PSD. Accurate pore sizes can only be obtained for reasonable porous structures and here, we only limit this calculation to densities of $0.5 \mathrm{~g} / \mathrm{cc}$ and $1.0 \mathrm{~g} / \mathrm{cc}$ structures. Carbon hybridization ( $s p, s p^{2}$ and $s p^{3}$ ) was computed from the number of nearest neighbor carbon atoms (2, 3 or 4 neighbors) within a cutoff radius of $1.73 \AA$. The above set of characterization tools form the basis for understanding the structural features of carbon as a function of density, simulation cell size, quench rate and annealing time.

\section{Results and discussion}


We explore the effects of four key simulation parameters viz. (a) structure density, (b) simulation cell size effect, (c) quench rate and (d) annealing time on the structural features of $a$ $C$. For each parameter, the range of values and other model characteristics like system size and density are outlined in Table 1.

\subsection{System density and its influence on $a-C$ formation}

Firstly, we study the structural features of $a-C$ at a range of densities from $0.5 \mathrm{~g} / \mathrm{cc}$ that mimics mesoporous char-like carbon, all the way up to $3.2 \mathrm{~g} / \mathrm{cc}$ mimicking diamond-like carbon (DLC) [5,31]. Figure 2 shows the atomic snapshots of the final structure for each density generated using a linear quench rate, $Q=33.3 \mathrm{~K} / \mathrm{ps}$ and annealed at 3,000 K for $140 \mathrm{ps.} \mathrm{For} \mathrm{this}$ study, the total number of atoms are set to $N=1200$, for all densities. The $0.5 \mathrm{~g} / \mathrm{cc}$ and $1.0 \mathrm{~g} / \mathrm{cc}$ structures are observed to be highly porous (with a porosity of $28 \%$ and $62 \%$ respectively). From $1.6 \mathrm{~g} / \mathrm{cc}$ onwards, the simulation cell is completely filled with carbon atoms rendering the structure non-porous. At $2.5 \mathrm{~g} / \mathrm{cc}$, we observe the structure is predominantly graphitic, with graphene-like sheets ordered nearly parallel to each with additional inter-planar bonds, in accordance with the results of both Saurez-Martinez et. al [32] who observed spontaneously evolution of graphite-like structures during high-temperature annealing for $a-C$ at similar density and Petersen et. al [26] who used the Reverse Monte Carlo method to model char at graphite-like density. At the largest density $(3.2 \mathrm{~g} / \mathrm{cc})$, the structure exhibits a rigid network of tetrahedrallycoordinated ( $s p^{3}$ hybridized) carbon atoms.

An important characterization for $a-C$ models is carbon hybridization. At low and intermediate densities (up to $\sim 2 \mathrm{~g} / \mathrm{cc}$ ), significant graphitization is typically observed $[13,32,33,53]$ that translates to significant $s p^{2}$ content. At larger densities, the closer packing of 
carbon atoms leads to increasing $s p^{3}$ hybridization. The final $s p^{2}$ and $s p^{3}$ hybridization content for $a-C$ at various densities considered in this work is presented in

Table 2. A direct comparison with other models from literature is also made.

Figure 3 shows the structural characterization of $a-C$ generated at various densities. The four sub-panels show (a) pair correlation functions, $g(r)$, (b) bond-angle distribution, (c) ring-size distribution and (d) percentage carbon hybridization. The pair correlation and bond-angle distribution functions are presented as stacked curves for clearer representation. The first peak in $\mathrm{g}(\mathrm{r})$ occurs at $1.44 \AA$ for the $0.5 \mathrm{~g} / \mathrm{cc}$, and it is approximately the same value till $2.5 \mathrm{~g} / \mathrm{cc}$. This corresponds to the $s p^{2}$ hybridization bond-length, which is the predominant hybridization for all these structures. For the $3.2 \mathrm{~g} / \mathrm{cc}$ structure, this peak occurs at $1.54 \AA$ and the peak is also broadened, owing to significant $s p^{3}$ hybridization. The second peak position for all the structures occurs around $2.5 \AA$, in agreement with models reported in literature. The low-density structures, notably $0.5 \mathrm{~g} / \mathrm{cc}$ and $1.0 \mathrm{~g} / \mathrm{cc}$ structures show a small shoulder at $\sim 1.2 \AA$, corresponding to the tiny fraction of 3-membered rings formed, which has also been observed in previous models [21,33]. The bond-angle distribution functions (panel b) are an alternate representation of the structural features. The bond angle ranges from $\sim 100^{\circ}$ to $140^{\circ}$ for densities up to $2.5 \mathrm{~g} / \mathrm{cc}$, with a peak at $120^{\circ}$. Thus, the predominant feature at these densities is six-membered graphene-like rings, with a small fraction of five and seven-membered rings. For all the structures described in this section, 3-dimensional coordinates in interactive 'xyz' visualization format are provided in supplementary material (pdf file titled 'part A'). 


\subsection{Simulation cell size effects}

We carry out a systematic study of the simulation cell size effects on structural features of $a-C$. At low densities, two possible scenarios could arise when the simulation cell size is increased - (a) the pore size could be invariant, with the spatial distribution of carbon atoms scaling with the increase in volume, and (b) the pore size could increase, with a greater clustering of atoms at larger volumes. To the best of our knowledge, such a study has not been performed in the literature. At larger densities, this effect is not important, or even meaningful, as the number density of carbon atoms is large enough to fill the simulation box that there are no welldefined pores possible.

In order to explore this effect, we vary the system size as $N=1,200,2,400,6,000,12,000$ and 30,000 atoms, at 3 densities, namely, $0.5 \mathrm{~g} / \mathrm{cc}, 1.0 \mathrm{~g} / \mathrm{cc}$ and $1.6 \mathrm{~g} / \mathrm{cc}$. The structures were linearly-quenched from $10,000 \mathrm{~K}$ to $3,000 \mathrm{~K}$ with a rate, $Q=33.3 \mathrm{~K} / \mathrm{ps}$ and annealed at 3,000 $\mathrm{K}$ for $140 \mathrm{ps}$. The final atomic snapshots for the structures are shown in Figure 4.

Structural characterization in terms of $\mathrm{g}(\mathrm{r})$, angles distribution and ring size distribution for the three densities are shown in Figure 5, panels (a), (b) and (c) respectively. We observe that for each density, there is negligible variation in the $\mathrm{g}(\mathrm{r})$ and bond angle distribution. With respect to structural features, we observe that six-membered rings are the most predominant (40-55\%) followed by five and seven-membered rings ( 12-16\% each). For each case, larger system sizes (most notably, $N=30,000)$ show an increase in six-membered ring formation $(\sim 10-30 \%$ increase) compared to the smallest size $(N=1,200)$. The hybridization content for all three

densities were also seen to be nearly identical, with $85-90 \% s p^{2}$ hybridization, and about $7 \% s p$ hybridization. This is shown in Figure 6 (a) for data averaged over different simulation cell sizes, for each of the three densities. However, a stark difference in pore sizes arises upon increasing 
simulation cell size, as shown in Figure 6 (b) for densities of $0.5 \mathrm{~g} / \mathrm{cc}$ and $1.0 \mathrm{~g} / \mathrm{cc}$. As seen from the figure, an increase in the simulation cell size results in an increase in the pore size. The maximum pore diameter was seen to increase more than two-fold (from $28.8 \AA$ for $N=1,200$ atoms, to about $64.3 \AA$ for $N=30,000$ atoms) for the $0.5 \mathrm{~g} / \mathrm{cc}$ case, and about 2.7 times (from 15 $\AA$ for $N=1,200$ atoms to about $41 \AA$ for $N=30,000$ atoms) for $1.0 \mathrm{~g} / \mathrm{cc}$ case. For densities from $1.6 \mathrm{~g} / \mathrm{cc}$ and larger, the density of the structure is large enough to render the concept of pore meaningless. The final structure at all system sizes, are, however, "fully-connected" in three dimensions. This characteristic dependence of pore size on system size is seen to be common for the liquid quench method in general, as we observed this phenomenon with the REBO [46] potential as well. The $N=30,000$ atom case with the REBO potential resulted in far greater clumping of atoms, where there was no periodicity along one of the simulation cell axes (not shown here).

We note that the differences in the structural features with system size exist only with respect to pore features. Figure 7 shows the evolution of carbon hybridization for $1.6 \mathrm{~g} / \mathrm{cc}$ carbon structure for various system sizes, during the entire liquid quench process (linear quench, followed by annealing at 3,000 K). We observe that the chemical evolution of the structure is nearly identical for all sizes, indicating that the only structural difference arising from the size effect is the pore size (as shown in Figure 6 (b)). We note that the invariance in the hybridization among different sizes was also observed for the $0.5 \mathrm{~g} / \mathrm{cc}$ and $1.0 \mathrm{~g} / \mathrm{cc}$ structures. 3-dimensional, interactive 'xyz' snapshots for all the sizes described above are provided in the supplementary material (listed as 3 pdf files titled 'part B-1', 'part B-2' and 'part B-3'). 


\subsection{Effect of quench rate}

Quench rate is another important parameter that affects structural features in liquid quench simulations. Typically, two kinds of quenching cycle have been employed in quench simulations in literature - linear (e.g. [28,53]) and exponential (e.g. [31,40]). In this work, we make a critical comparison of structural evolution via both quenching routes for $a$ - $C$ consisting of $N=1,200$ atoms, at a density of $1.0 \mathrm{~g} / \mathrm{cc}$.

\subsubsection{Linear quench}

The linear quench rate, $Q=d T / d t$ was varied from $11.1 \mathrm{~K} / \mathrm{ps}$ to $100 \mathrm{~K} / \mathrm{ps}$ while quenching from a temperature of $10,000 \mathrm{~K}$ to $3,000 \mathrm{~K}$, preceding the annealing stage. Figure 8 shows the structural evolution including (a) the $\mathrm{g}(\mathrm{r})$, (b) bond angle distribution, (c) ring size distribution and (d) pore size distribution for the different quench rates, immediately after the quench stage. Data have been averaged over three independent samples for each quench rate. As can be seen, both pair correlation functions and bond angle distribution are nearly invariant with quench rate. The percentage of three-membered rings is, however, quite significant at higher quench rates, evident from both the peak at $\sim 60^{\circ}$ in the bond angle distribution (panel b) and the ring size distribution (panel c). The large fraction of three-membered rings demonstrates that the structure is still in a transition-phase towards graphitization at higher quench rates. We also observe that probabilistically, it is easier to form three-membered rings (which are, in fact, very high-energy configurations) at larger quench rates, that transition to six-membered rings with subsequent annealing. The pore size distribution (panel (d)), shows a sharp increase in peak positions (signifying larger pores) when $Q$ is increased decreased from $100 \mathrm{~K} / \mathrm{ps}$ to $50 \mathrm{~K} / \mathrm{ps}$, followed by a saturation of pore sizes at lower $Q$. This initial increase in pore size with decreasing quench rate is consistent with the observation of Shi [33] who used the RSS potential 
to model three structures of amorphous carbon at densities of $0.76 \mathrm{~g} / \mathrm{cc}, 1.0 \mathrm{~g} / \mathrm{cc}$ and $1.5 \mathrm{~g} / \mathrm{cc}$ and observed that by increasing the quench rate, the pore size decreases on average.

Figure 9 shows the evolution of carbon hybridization (averaged over three independent samples) with quench rate. The variation of hybridization between each independent sample for any given quench rate was observed to be negligibly small. As seen from the figure, a smaller quench rate gives rise to a larger fraction of $s p^{2}$ hybridization (and consequently, greater graphitization) as a result of more time for the system to get ordered. The relatively large

difference in the $s p^{2}$ content (difference of $\sim 10 \%$ ) upon lowering the quench rate plays an important role in determining the final carbon hybridization ratios in the subsequent annealing stage and the final structure.

\subsubsection{Exponential quench}

Exponential quenching is another commonly used quench setup in literature $[31,40]$. In this work, various exponential quench rates are achieved by varying the exponential factor, $A$, according to eq [1].

$$
T(K)=7000 * \exp (-A t)+3000
$$

The temperature ranges from 10,000 to $3,000 \mathrm{~K}$, which are the same values as for the linear quench. The total time for the quench is set as $210 \mathrm{ps}$ (note that this time corresponds to the time for a linear quench rate of $Q=33.3 \mathrm{~K} / \mathrm{ps}$ ). We consider six quenching exponents, $A$, ranging from $A=1.5 \times 10^{10}$ to $A=6 \times 10^{11}$. Figure 10 shows the structural evolution akin to the analysis for the linear quench. Similar to linear quench, we observe negligible variation in the $\mathrm{g}(\mathrm{r})$ and bond angle distribution curves for different exponential quench rates. In addition, the ring size distribution (panel (c)) is also found to be quench-rate independent, with a predominant 
fraction of six-membered rings (ranging from $\sim 36 \%$ for the smallest quench rate to $\sim 52 \%$ for the largest rate) and about $15 \%$ of each of five and seven-membered rings. This relatively rapid evolution of the structure is a direct consequence of the exponential nature of quench, where, a temperature close to $3,000 \mathrm{~K}$ is reached far sooner compared to the linear quench case, and naturally, the system has more time to reach a more stable configuration. Pore size distribution analysis (panel (d)) is seen to exhibit a small increase in pore size with decrease in quench rate, followed by a saturation. We suppose that the much weaker dependence between pore size and quench rate, as compared to the linear quench also has its origin in the exponential nature of quench; once the temperature decreases exponentially (to $\sim 3,000 \mathrm{~K}$ ), the system evolves under an approximately isothermal condition for all quench rates.

Figure 11 shows the evolution of carbon hybridization as a function of the exponential quench rate. A faster quench rate results in more rapid $s p^{2}$ hybridization conversion (which leads to more graphitization). Based on the same reasoning of rapid decay of temperature to close to $3,000 \mathrm{~K}$ for the exponential case, the structural evolution is observed to be similar for all quench rates except the two lowest rates $\left(A=1.5 \times 10^{10}\right.$ and $\left.3 \times 10^{10}\right)$. For larger rates, a saturation behavior is observed, and a very high percentage of $s p^{2}$ hybridization (85-88\%) results at the end of the quench stage.

\subsection{Effect of annealing time}

A key step in the liquid quench method is the annealing stage, immediately after the quench stage, where the structure is "annealed" for a given amount of time, typically at high temperatures. This step is essential to convert the high-energy structure from the quench stage to 
a relaxed and equilibrated (at the anneal temperature) one at the end of annealing stage. To explore the effect of annealing time and the corresponding structural changes brought about during this step, we perform a long annealing simulation for $1.0 \mathrm{~g} / \mathrm{cc} a-C$, for an anneal time, $t_{a}$ of 340 ps. Figure 12 shows the evolution of carbon hybridization as a function of anneal time, averaged over two independent samples. As seen from the figure, significant structural evolution is seen to take place till $\sim 200 \mathrm{ps}$, after which the hybridization ( $s p$ and $s p^{2}$ ) exhibits a plateau. The maximum $s p^{2}$ fraction is observed to be $\sim 94 \%$, signifying extensive ordering of the structure via graphitization.

In Figure 13, we show the evolution of carbon hybridization during the annealing stage for various quenched structures, using both linear quench (panel (a)) and exponential quench (panel (b)). We observe that the total $s p^{2}$ content at the end of annealing stage ( $\left.140 \mathrm{ps}\right)$ varies between $84 \%$ (highest quench rate) to $90 \%$ (lowest quench rate) for linear quench and is about $90 \%$ for all exponentially quenched samples. A larger $s p^{2}$ is attained more quickly for exponential quench compared to linear quench owing to the nature of temperature profile as mentioned before. Thus, to obtain a well-ordered structure (large $s p^{2}$ fraction) via the linear quench route, significant annealing times (of the order of 150 ps or more) would be necessary.

\subsection{Perspectives on generated $a-C$ models}

\subsubsection{Comparison with models in literature}

In this section, we compare the generated $a-C$ models with experimental results for pair correlation functions $(\mathrm{g}(\mathrm{r}))$ and pore size distributions, where available [21,22,24,27,58-61]. Pair correlation functions are typically measured experimentally by diffraction techniques, including 
X-ray and electron diffraction. Pore sizes are typically measured experimentally via adsorption of a gas such as argon. Shown in Figure 14 are the pair correlation data for $a-C$ for various densities ranging from $1 \mathrm{~g} / \mathrm{cc}$ to $3.2 \mathrm{~g} / \mathrm{cc}$ generated using the liquid quench route, compared with experimental results. We observe that the peak positions and heights agree well for almost all cases, signifying realistic short-range order in these structures. Pore size distributions for $1 \mathrm{~g} / \mathrm{cc}$ $a-C$ generated via both linear and exponential quench routes (refer to section 3.3) are compared with data from experiments and a HRMC model from literature, in Figure 15. For the quench results (linear and exponential), data are averaged across multiple samples and across various quench rates as described in section 3.3. A reasonably wide spread of pore sizes is observed via the liquid quench route, owing to the stochastic nature of the quench process. However, we do observe that the range of pore sizes matches reasonably well with experimental measurements.

\subsubsection{Comparison with $\operatorname{ReaxFF}_{C-2015}$ potential}

All the simulations reported here so far correspond to an early version of $R e a x F F_{C H O}$ potential for hydrocarbons published by Chenoweth et. al. in 2008 [48]. In this section, we compare several test cases of $a-C$ generated with different simulation conditions with a more recent ReaxFF parametrization developed by Srinivasan et. al., henceforth referred to as $\operatorname{ReaxFF}_{\mathrm{C}-2015}$ [62]. Specifically, we quantify structural features as described in previous sections for $a-C$ at densities of $1 \mathrm{~g} / \mathrm{cc}$ and $2 \mathrm{~g} / \mathrm{cc}$ (generated using a linear quench rate); size effect for 1 $\mathrm{g} / \mathrm{cc} a-C$ (linear quench rate), and for two exponential quench rates, namely $Q=3 \times 10^{10}$ and $Q=$ $3 \times 10^{11}$ generated using the $\operatorname{ReaxFF}_{\mathrm{C}-2015}$ and compare with those obtained using $R e a x F F_{C H O}$. These details are listed in Table 3, and, in addition, 'xyz' snapshots for all these structures generated using $\operatorname{ReaxFF}_{\mathrm{C}-2015}$ are provided in the supplementary material (listed as pdf file titled 
'part C'). Common structural features as described in previous sections were quantified for $a-C$ generated using both versions of the ReaxFF potential and are compared in Figure 16. As seen from the figure, except for small differences, both potentials give rise to very similar structural features. Specifically, we observe that minor differences include - ReaxFF $F_{C-2015}$ results in lesser number of 3-membered rings (see panel (b)), larger pore sizes (panel (e)), and marginally faster evolution of $s p^{2}$ hybridization compared to ReaxFF $F_{C H O}$. However, the qualitative trends observed with $\operatorname{ReaxFF}_{C H O}$ are seen to hold for $\operatorname{ReaxFF}_{C-2015}$ as well, namely, (a) the observed system size effect (i.e. larger simulation cell size resulting in larger pore sizes) as seen in panel (e) comparing pore sizes for $N=1,000$ atoms, and $N=6,000$ atoms, and (b) larger quench rate during exponential quench leads to faster evolution of $s p^{2}$ hybridization (panel (d)). Note that in Figure 16, PSD for $2 \mathrm{~g} / \mathrm{cc}$ structure is ill-defined due to the large density, and hence this is omitted in panel (e).

\section{Conclusions}

We have demonstrated the capability of modeling amorphous carbon $(a-C)$ at a wide range of densities ranging from $0.5 \mathrm{~g} / \mathrm{cc}$ to $3.2 \mathrm{~g} / \mathrm{cc}$ using the highly-detailed ReaxFF reactive force field and the liquid quench method. An extensive characterization of structural features, including pair correlation, bond angle distribution, ring size distribution, carbon hybridization content and pore size distribution have been performed, and have been found to be consistent with previous models reported in literature. Our key findings are:

1. A large range of densities (from $0.5 \mathrm{~g} / \mathrm{cc}$ to $3.2 \mathrm{~g} / \mathrm{cc}$ ) can be modeled satisfactorily using the liquid quench method. For densities up to $2.5 \mathrm{~g} / \mathrm{cc}$, the structures exhibit a high fraction of $s p^{2}$ 
hybridized carbon ( $87 \%$ to $92 \%$ ) and predominantly six-membered rings. For a density of 3.2 $\mathrm{g} / \mathrm{cc}$, we observe about $45 \% s p^{2}$ and $52 \% s p^{3}$ hybridization.

2. Simulation box size effects play an important role in structural features, most notably for pore sizes for low-density structures $(0.5 \mathrm{~g} / \mathrm{cc}$ and $1.0 \mathrm{~g} / \mathrm{cc}$ in this work). We find that larger simulation cell sizes result in structures with larger pores.

3. Quench rate is seen to play an important role in the evolution of structural features. For $a-C$ at $1.0 \mathrm{~g} / \mathrm{cc}$, we observe that an exponential quench route results in a structure that contains a highly ordered, graphitic structure (close to $90 \% s p^{2}$ hybridized carbon). Linear quenching, on the other hand leads to a much lower $s p^{2}$ fraction at the end of quench stage, which necessitates long annealing times of the order of $150 \mathrm{ps}$ or more to obtain significant $s p^{2}$ hybridization (graphitization). Increasing quench rate is also seen to, in general, result in smaller pore sizes.

4. Annealing the structure after the quench stage relaxes the structure further and is observed to cause a more ordered structure with further increase in $s p^{2}$ content. For linear quench, larger annealing times maybe necessary to obtain the same amount of structural ordering, as compared to an exponential quench.

5. A critical comparison between the generated $a-C$ models with experimental data published in literature was made. We observed that pair correlation functions of the generated models agree well with experiments, for a wide range of $a-C$ at various densities. Additionally, comparison of generated models with those generated via a newer ReaxFF implementation for condensed carbon phases, named $R e a x F F_{C-2015}$ yielded qualitatively similar structural features.

Using the liquid quench method with ReaxFF, it is thus possible to generate a wide variety of amorphous carbon structures with a range of chemical and structural features. One can, 
in principle, choose a specific synthesis condition to come up with an appropriate model that mimics a physical $a-C$ material.

\section{Acknowledgements}

This work was sponsored by Naval Air Systems Command (NAVAIR) through a Small Business Technology Transfer (STTR) grant, contract no. N68335-13-C-0119, awarded to Advanced Cooling Technologies (ACT), Inc., Lancaster, Pennsylvania and was performed at ACT [39]. The authors would like to thank technical advice of Peter Cross and Richard Burnes, from Naval Air Warfare Center Weapons Division (NAWCWD) - China Lake, CA. In addition thanks are also extended to Prof. Donald Brenner at North Carolina State University for valuable suggestions.

The modeling efforts have benefited from computing time provided by NAWCWD on Spirit (AFRL) supercomputer. Most of the production simulations reported in this work have been undertaken using high-performance computing infrastructure of Extreme Science and Engineering Discovery Environment (XSEDE) [63], provided to Dr. Srujan Rokkam (ACT) under allocation grant TG-SBR150001. XSEDE is supported by National Science Foundation (NSF) grant number ACI-1053575. 
Table 1: List of various simulation parameters in modeling amorphous carbon.

\begin{tabular}{|c|c|c|}
\hline Effect & Range of values & $\begin{array}{c}\text { System size (number of } \\
\text { atoms, } \boldsymbol{N} \text { ) and density }\end{array}$ \\
\hline Density & $\begin{array}{c}0.5,1.0,1.6,2.02 .5 \text { and } \\
3.2 \mathrm{~g} / \mathrm{cc}\end{array}$ & $\begin{array}{c}N=1,200 ; \text { various } \\
\text { densities }\end{array}$ \\
\hline Size effect & $\begin{array}{c}N=1,200,2,400,6,000, \\
12,000 \text { and } 30,000 \text { atoms }\end{array}$ & $1.0 \mathrm{~g} / \mathrm{cc}$ \\
\hline $\begin{array}{c}\text { Linear quench rate, } \\
Q\end{array}$ & $\begin{array}{c}11.1,22.2,33.3,50 \text { and } \\
100 \mathrm{~K} / \mathrm{ps}\end{array}$ & $N=1,200,1.0 \mathrm{~g} / \mathrm{cc}$ \\
\hline $\begin{array}{c}\text { Exponential quench } \\
\text { rate, } A\end{array}$ & $\begin{array}{c}1.5 \times 10^{10}, 3 \times 10^{10}, 6 \times 10^{10} \\
1.2 \times 10^{11}, 3 \times 10^{11} \text { and } \\
6 \times 10^{11}\end{array}$ & $N=1,200,1.0 \mathrm{~g} / \mathrm{cc}$ \\
\hline Annealing time & $\mathrm{t}=340 \mathrm{ps}$ & $N=1,200,1.0 \mathrm{~g} / \mathrm{cc}$ \\
\hline
\end{tabular}

Table 2: Final $s p^{2}$ and $s p^{3}$ content for various densities of amorphous carbon in this study. Hybridization content for similar densities from literature are given for comparison.

\begin{tabular}{|c|c|c|c|c|}
\hline $\begin{array}{c}\text { Density } \\
(\mathrm{g} / \mathrm{cc})\end{array}$ & $\begin{array}{c}\text { Final } \boldsymbol{s} \boldsymbol{p}^{\mathbf{2}} \\
\text { content }(\%)\end{array}$ & $\begin{array}{c}\text { Final } \boldsymbol{s} \boldsymbol{p}^{\mathbf{2}} \text { content } \\
\text { (literature) }\end{array}$ & $\begin{array}{c}\text { Final } \boldsymbol{s} \boldsymbol{p}^{\mathbf{3}} \\
\text { content }(\%)\end{array}$ & $\begin{array}{c}\text { Final } \boldsymbol{s} \boldsymbol{p}^{3} \text { content } \\
\text { (literature) }\end{array}$ \\
\hline 0.5 & 87.4 & - & 1.7 & - \\
\hline 1.0 & 79.5 & $66-80[24]$ & 1.3 & $1-5[24]$ \\
\hline 1.6 & 89.0 & $90-98[32]$ & 1.8 & $0.2-7[32]$ \\
\hline
\end{tabular}




\begin{tabular}{|c|c|c|c|c|}
\hline 2.0 & 91.7 & $\begin{array}{c}\{36,75,82 \\
[40]\}^{*}\end{array}$ & 2.7 & $\begin{array}{c}15[31,40] ;\{20, \\
30,60-70[40]\}^{*}\end{array}$ \\
\hline 2.5 & 92.2 & $\{25,70,80[40]\}$ & 5.2 & $\begin{array}{c}20,30[31] ;\{10, \\
20,70[40]\}\end{array}$ \\
\hline 3.2 & 44.6 & $\begin{array}{c}15-21[8] ;\{18, \\
43,80[40]\}\end{array}$ & 52 & $\begin{array}{c}\{18,50,82[40]\} ; \\
60[31] ;\{76-80 \\
[8]\}\end{array}$ \\
\hline
\end{tabular}

* Structure is of density $2.2 \mathrm{~g} / \mathrm{cc}$. Ref [40] involves comparison of Tersoff, REBO and ReaxFF force fields for generating model $a-C$ at various densities.

Table 3: Simulation parameters for comparison of $a-C$ structures generated using $R e a x F F_{C H O}$ and $\operatorname{ReaxFF}_{\mathrm{C}-2015}$ potentials.

\begin{tabular}{|c|c|c|}
\hline System & System size & Quench rate \\
\hline $1 \mathrm{~g} / \mathrm{cc}-$ Linear quench & $\mathrm{N}=1,200$ and \\
6,000 & $\begin{array}{c}\text { Quench } \text { rate }=33.3 \\
\text { K/ps }\end{array}$ \\
\hline $2 \mathrm{~g} / \mathrm{cc}-$ Linear quench & $\mathrm{N}=1,200$ & \begin{tabular}{c} 
Quench rate $=33.3 \mathrm{~K} / \mathrm{ps}$ \\
\hline $1 \mathrm{~g} / \mathrm{cc}-$ Exponential quench
\end{tabular} \\
$\mathrm{N}=1,200$ & $\begin{array}{c}\text { Quench } \text { rate }=3 \times 10^{10} \\
\text { and } 3 \times 10^{11}\end{array}$ \\
\hline
\end{tabular}




\section{List of Figures}

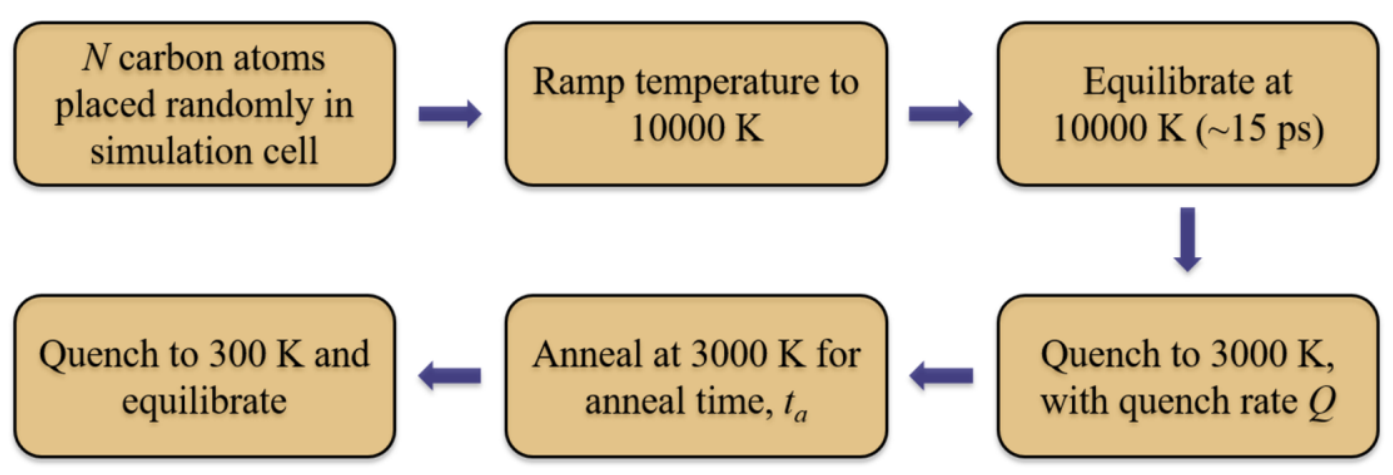

Figure 1: Flowchart of the liquid quench simulation process to generate amorphous carbon 

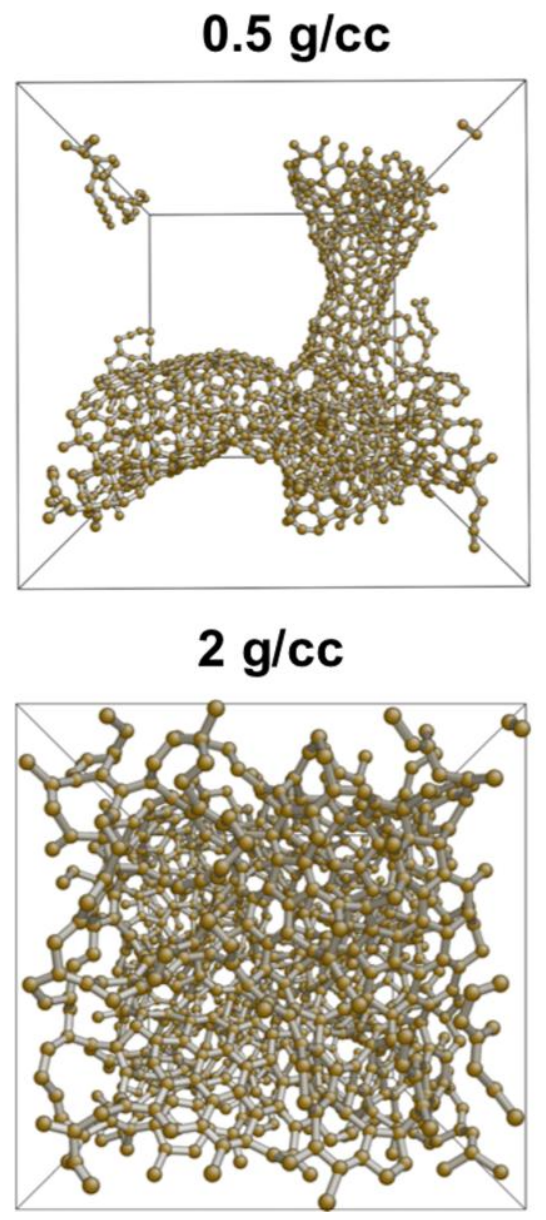

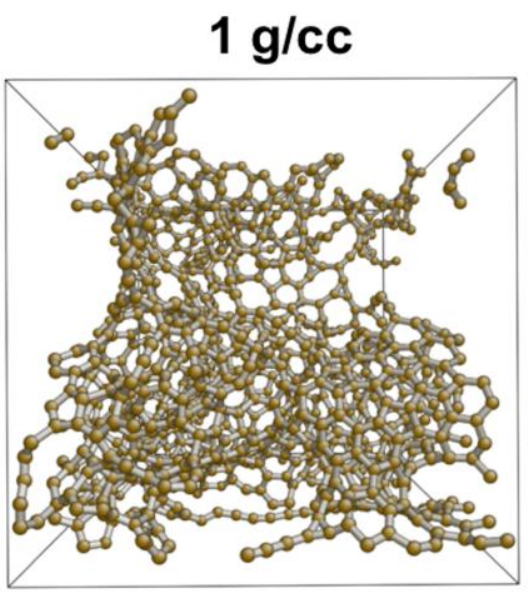

$2.5 \mathrm{~g} / \mathrm{cc}$

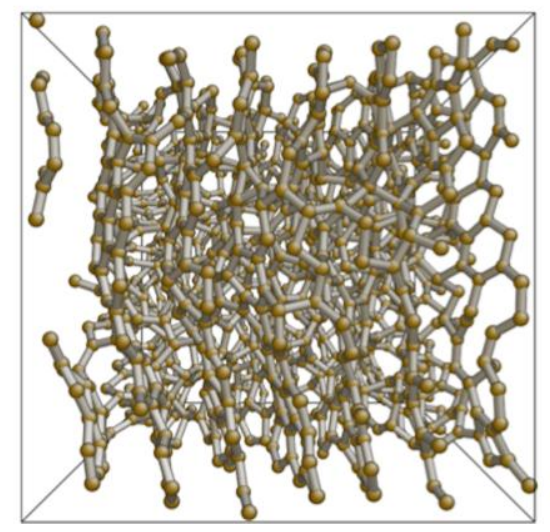

$1.6 \mathrm{~g} / \mathrm{cc}$

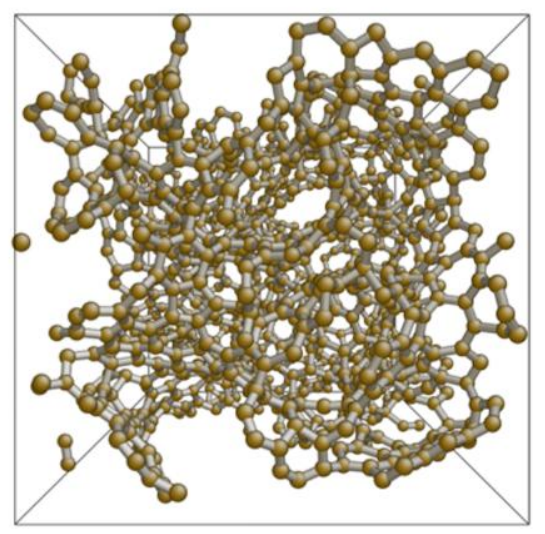

$3.2 \mathrm{~g} / \mathrm{cc}$

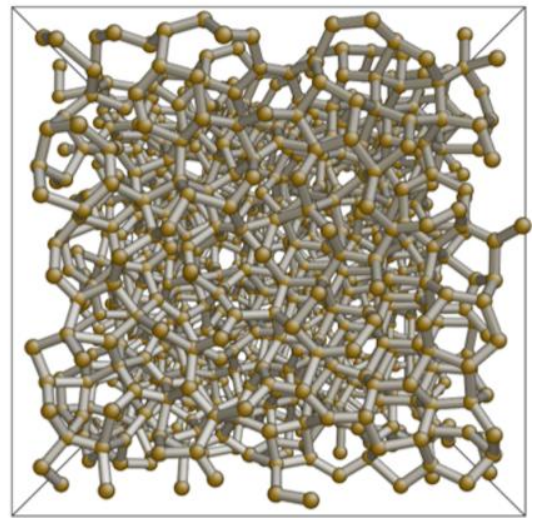

Figure 2: Final atomistic snapshots of amorphous carbon at various densitites ranging from 0.5 $\mathrm{g} / \mathrm{cc}$ to $3.2 \mathrm{~g} / \mathrm{cc}$. All strucrures contain $N=1200$ atoms. Refer to supplementary material for 3-D 'xyz' coordinates of all the structures. 
(a)

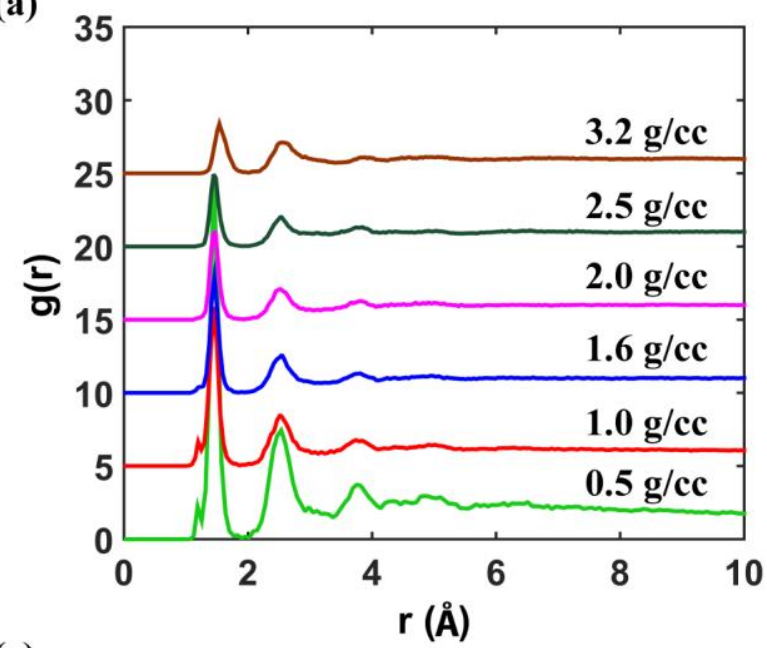

(c)

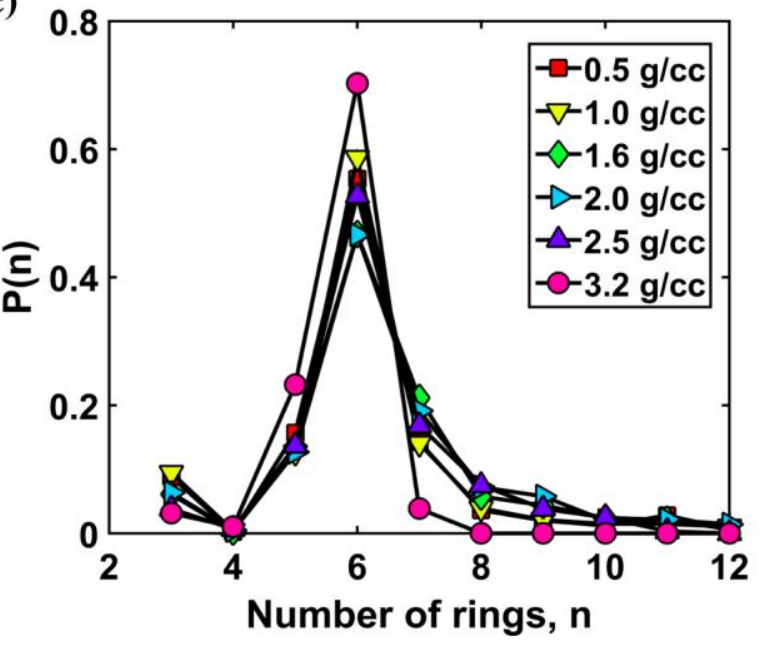

(b)

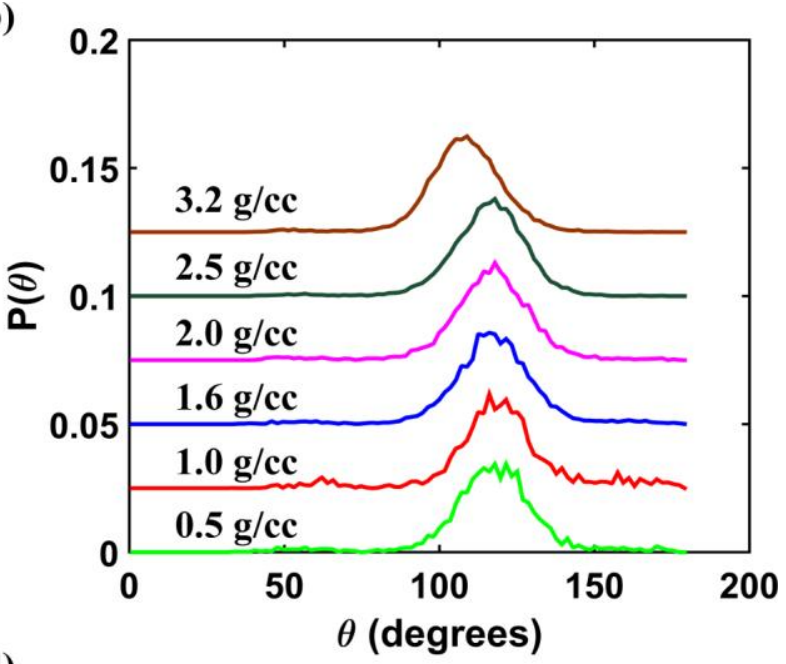

(d)

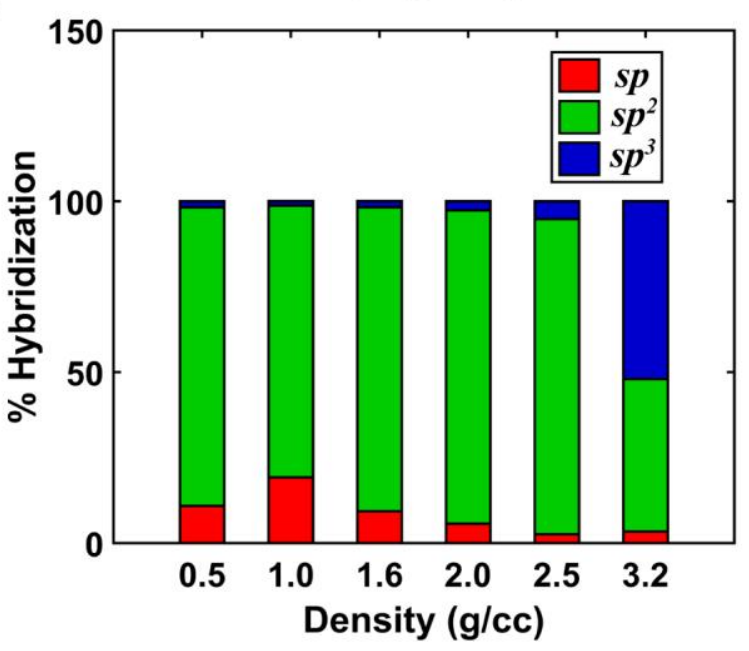

Figure 3: Structural characterization of amorphous carbon generated at various densities. (a) Pair correlation functions, (b) bond-angle distribution, (c) ring-size distribution and (d) percentage hybridization. The pair correlation and bond-angle distribution functions are presented as stacked curves for clearer representation. 


\section{$0.5 \mathrm{~g} / \mathrm{cc}$}
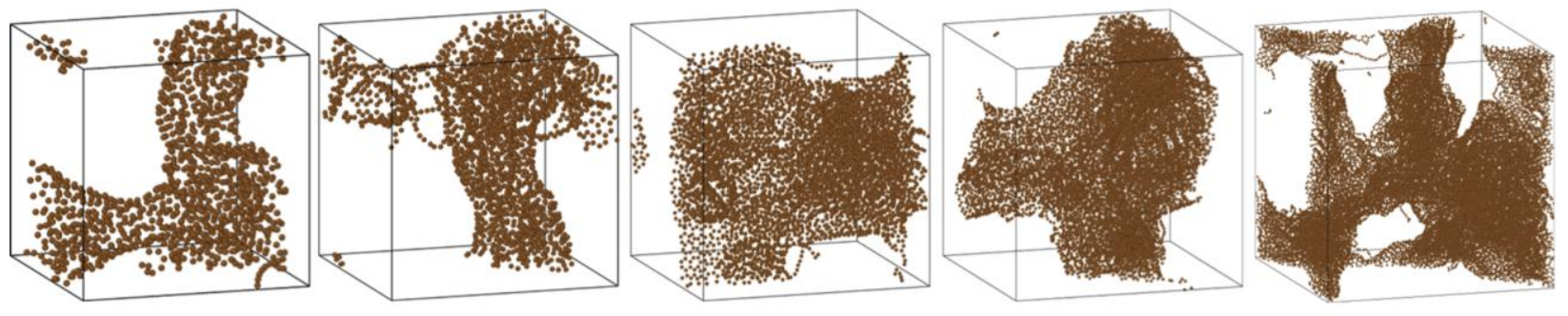

\section{$1 \mathrm{~g} / \mathrm{cc}$}
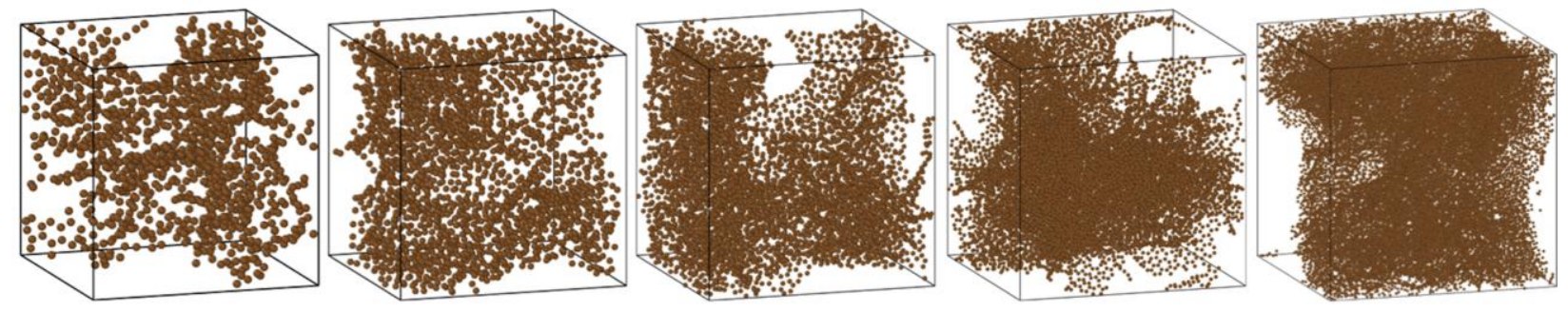

$1.6 \mathrm{~g} / \mathrm{cc}$
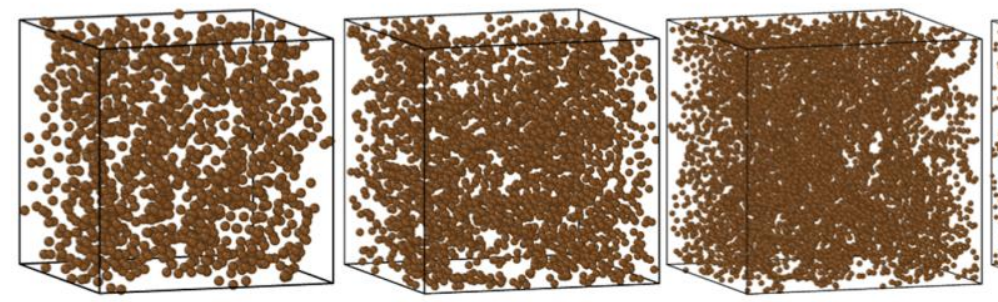

$N=1200$

$N=2400$

$N=6000$

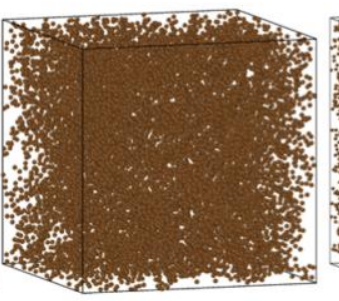

$N=6000$

$N=30000$

Figure 4: Final atomistic snapshots of amorphous carbon at three densities (in the low-density regime), namely, $0.5 \mathrm{~g} / \mathrm{cc}, 1.0 \mathrm{~g} / \mathrm{cc}$ and $1.6 \mathrm{~g} / \mathrm{cc}$ to depict simulation-cell size effects. The lowest densities $(0.5 \mathrm{~g} / \mathrm{cc}$ and $1.0 \mathrm{~g} / \mathrm{cc})$ show significant size effects, with larger cell sizes leading to correspondingly larger pore sizes. The number of atoms $(N)$ range from $N=1,200$ to $N=30,000$. Refer to supplementary material for 3-D 'xyz' coordinates of all the structures. 

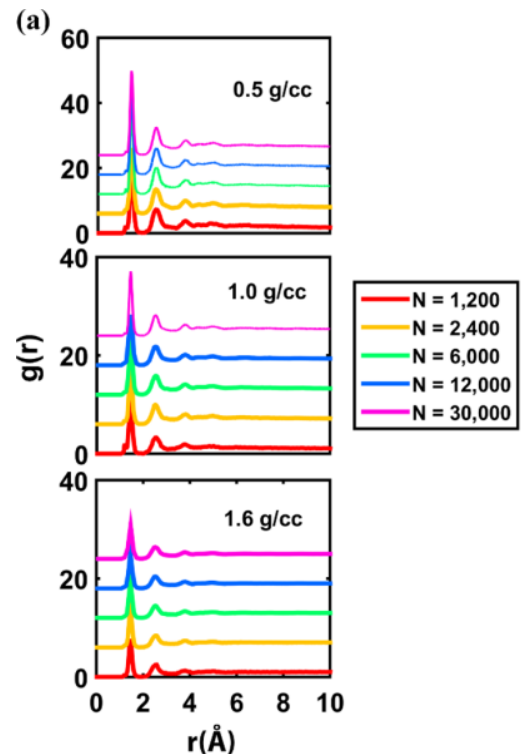
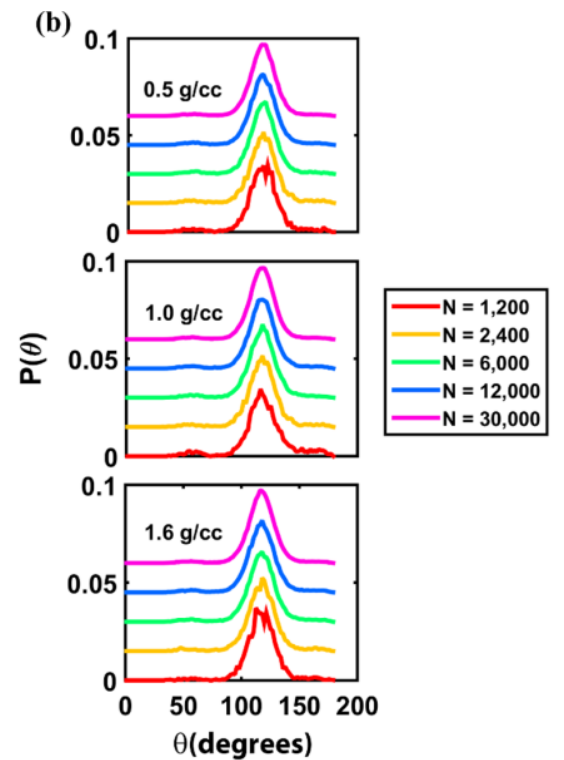

(c)
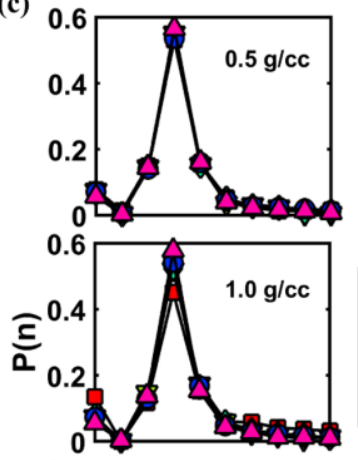

$-\square-N=1,200$

$-\nabla-N=2,400$ $\begin{aligned}-N & =6,000 \\ -N & =12,000\end{aligned}$ $\triangle-N=30,000$

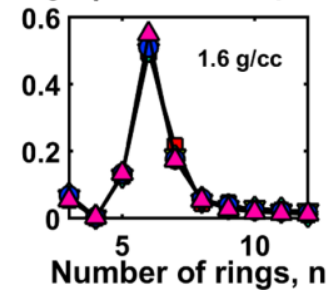

Figure 5: Structural characterization of amorphous carbon for three different densities, $0.5 \mathrm{~g} / \mathrm{cc}$, $1.0 \mathrm{~g} / \mathrm{cc}$, and $1.6 \mathrm{~g} / \mathrm{cc}$, for various system sizes $N$. 

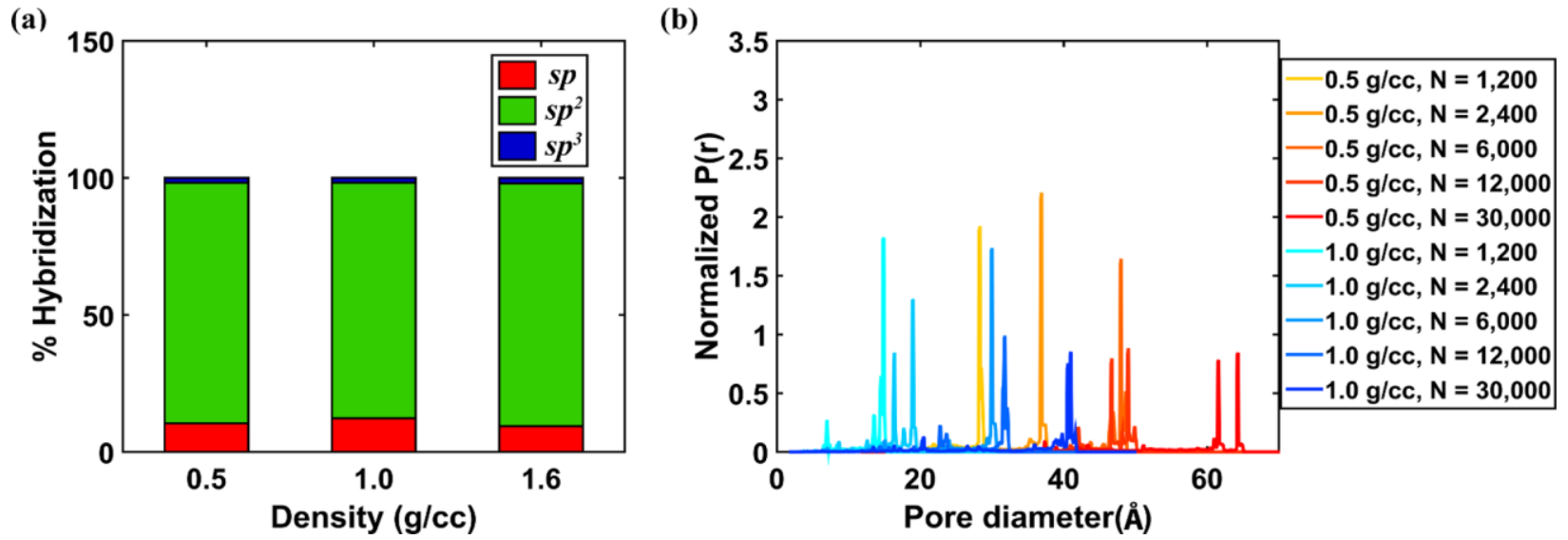

Figure 6: (a) Averaged final hybridization content for different densities in the low density regime and (b) pore size distribution for various simulation cell sizes for $0.5 \mathrm{~g} / \mathrm{cc}$ and $1.0 \mathrm{~g} / \mathrm{cc}$ structures. 

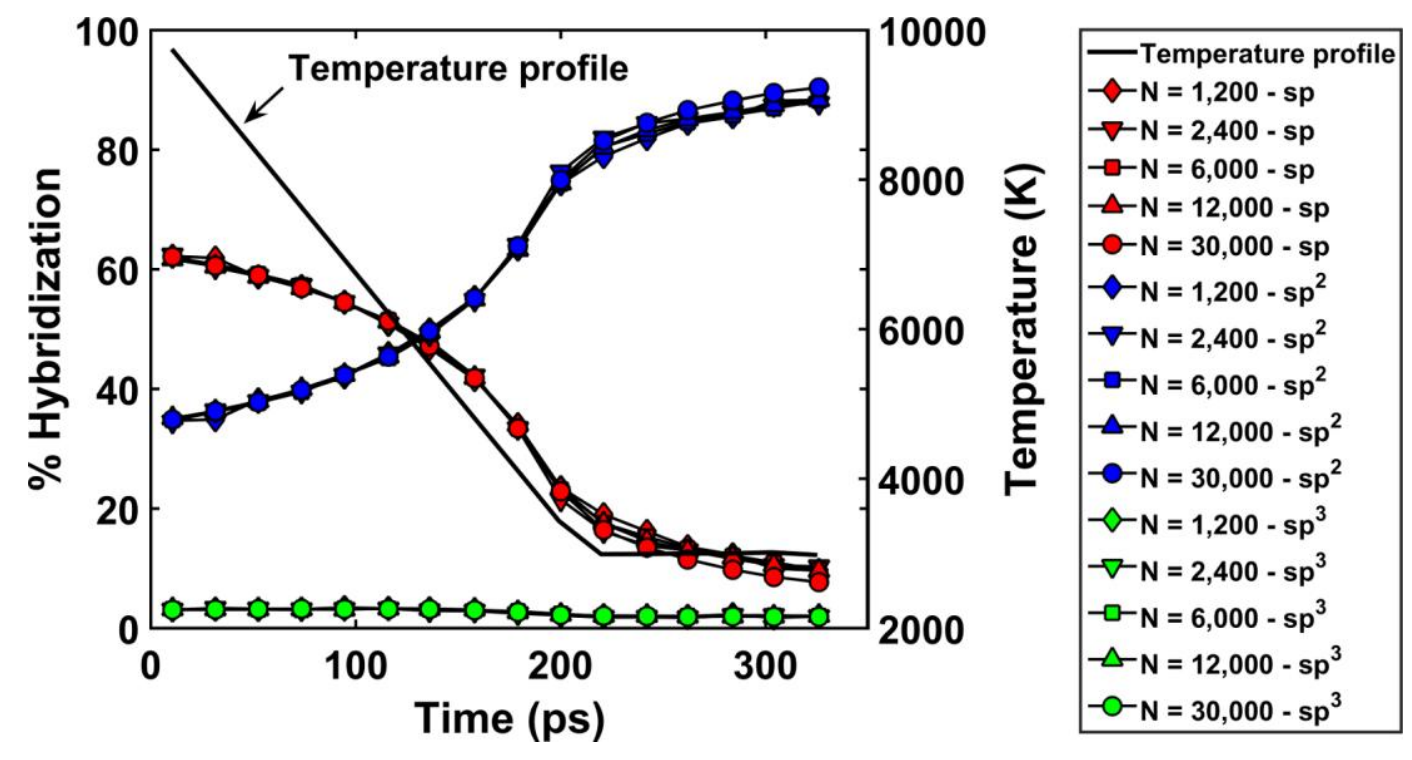

Figure 7: Variation of carbon hybridization content for various simulation cell sizes during the liquid quench process, for $1.6 \mathrm{~g} / \mathrm{cc}$ carbon structure. The temperature profile during linear quench, followed by annealing at 3,000 $\mathrm{K}$ is shown by black solid line. 
(a)

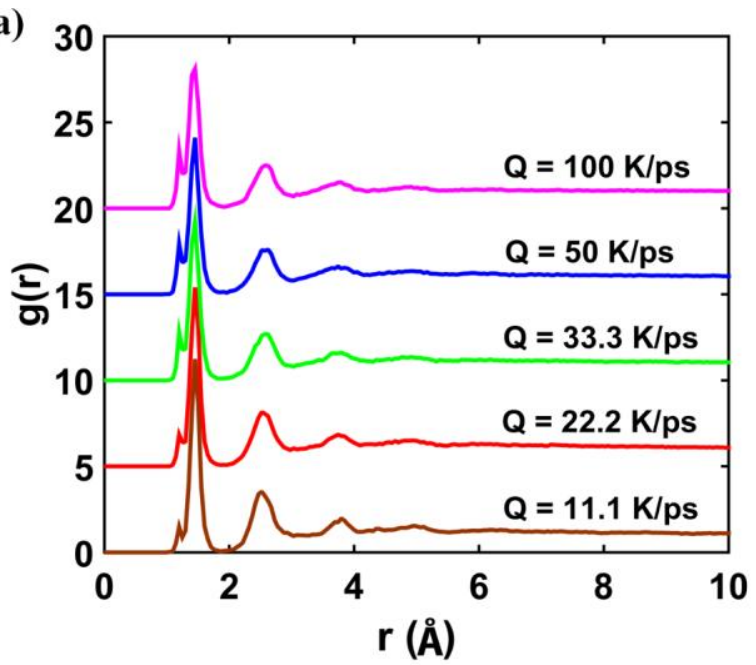

(c)

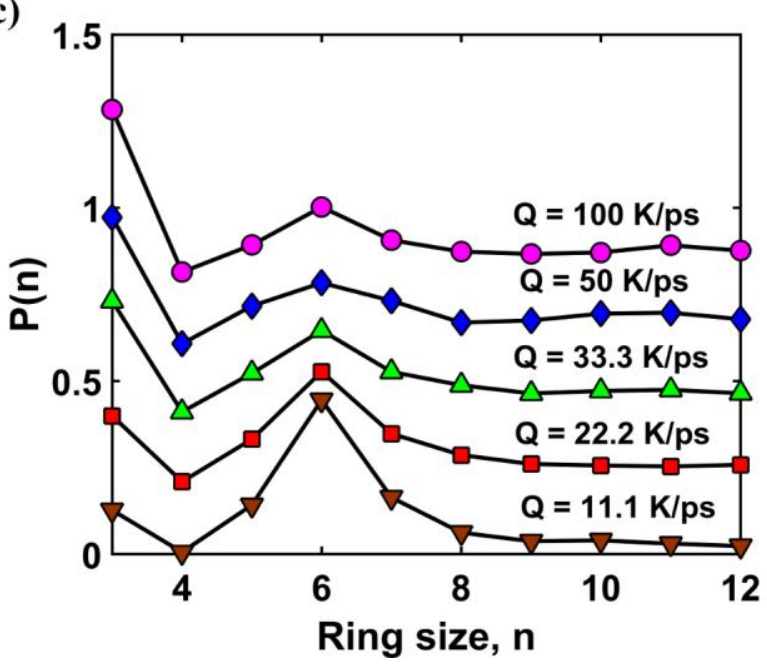

(b)

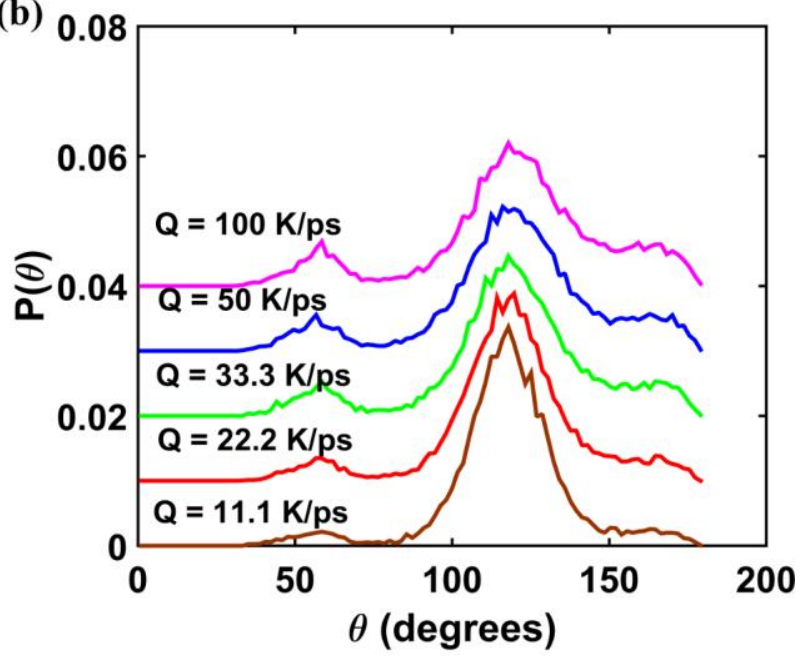

(d)

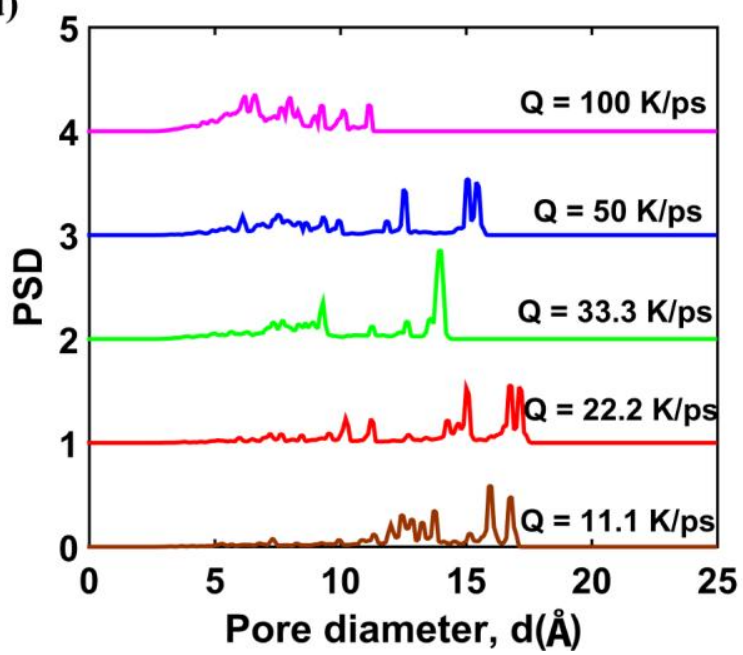

Figure 8: Structural characterization of amorphous carbon at $1.0 \mathrm{~g} / \mathrm{cc}$ for various quench rates at the end of linear quench stage. (a) Pair correlation functions, (b) bond-angle distribution, (c) ring-size distribution, and (d) pore size distribution (PSD). Data are averaged over 4 samples for each quench rate. 

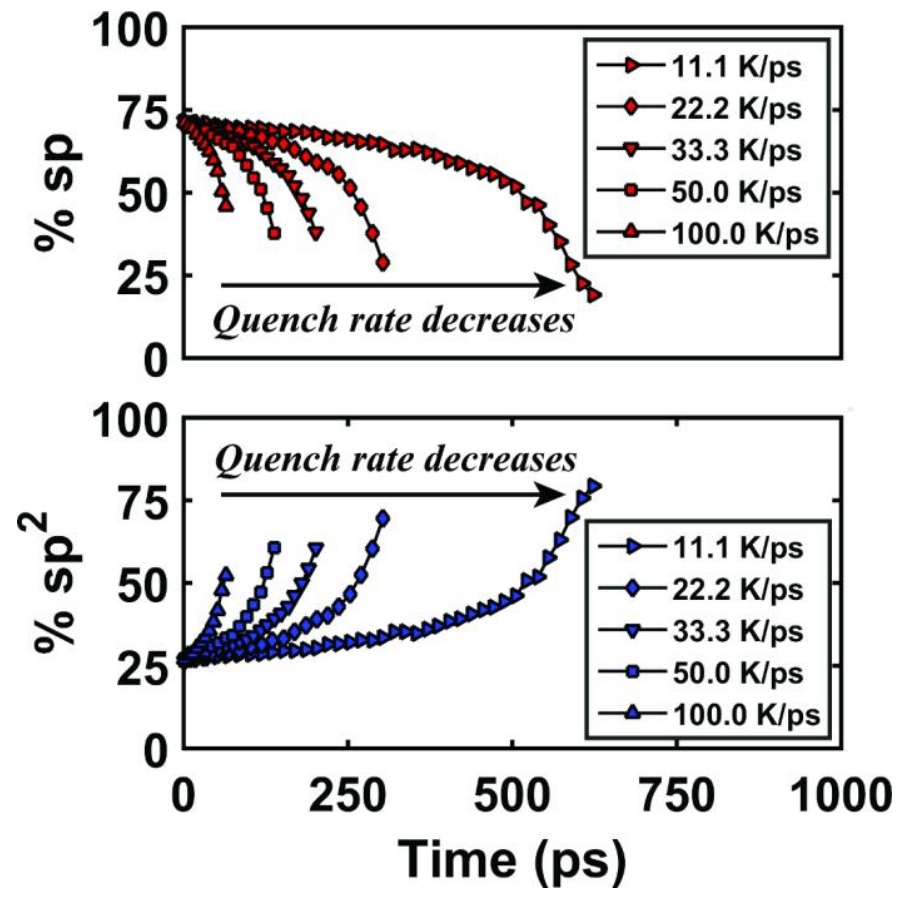

Figure 9: Variation of carbon hybridization during linear quenching for different quench rates.

The $s p^{3}$ content is negligible for all cases $(<2 \%)$ and is not shown. 

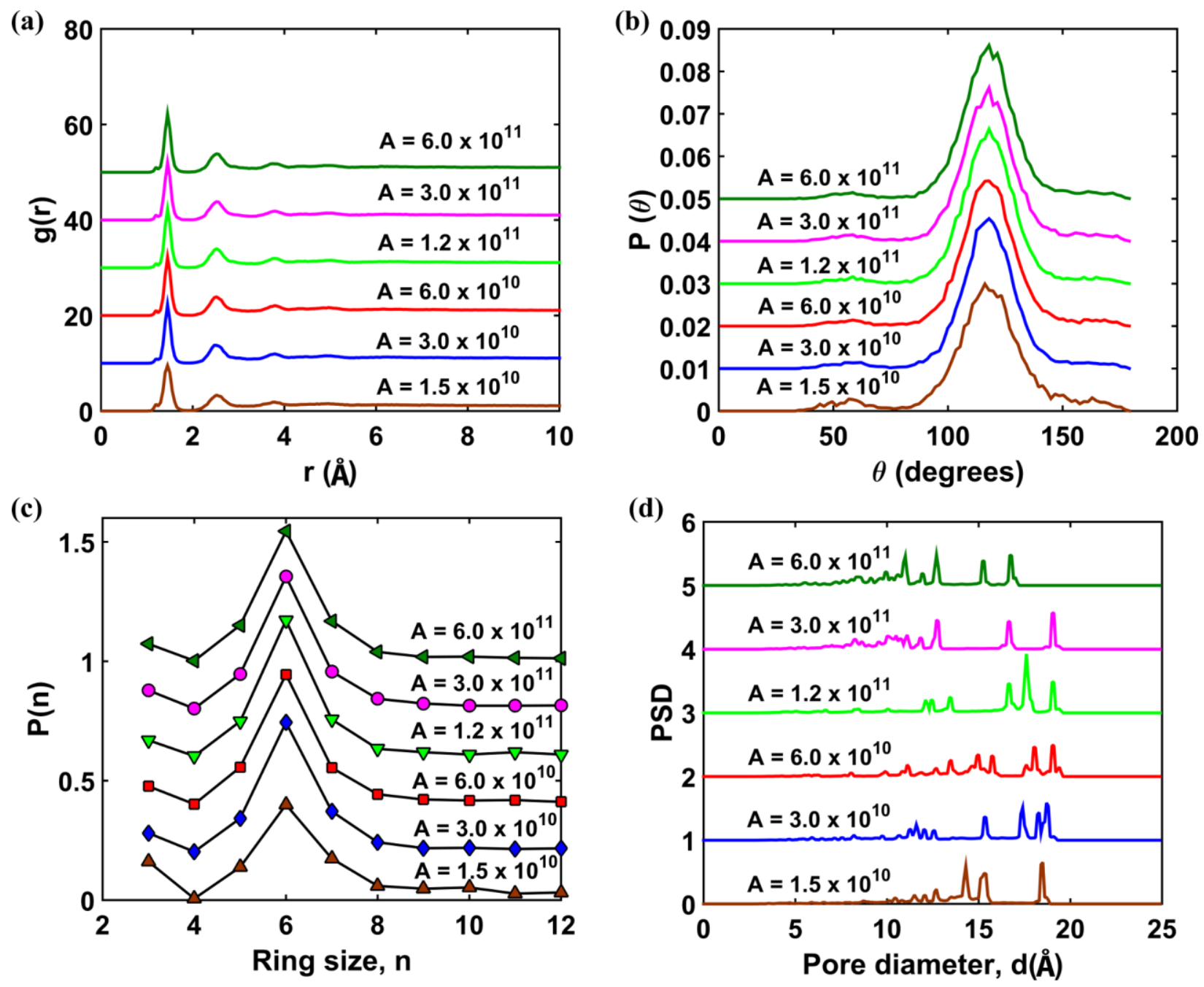

Figure 10: Structural characterization of amorphous carbon at $1.0 \mathrm{~g} / \mathrm{cc}$ for various quench rates using an exponential quench step. (a) Pair correlation functions, (b) bond-angle distribution, (c) ring-size distribution, and (d) pore size distribution (PSD). Data are averaged over 4 samples for each quench rate. 

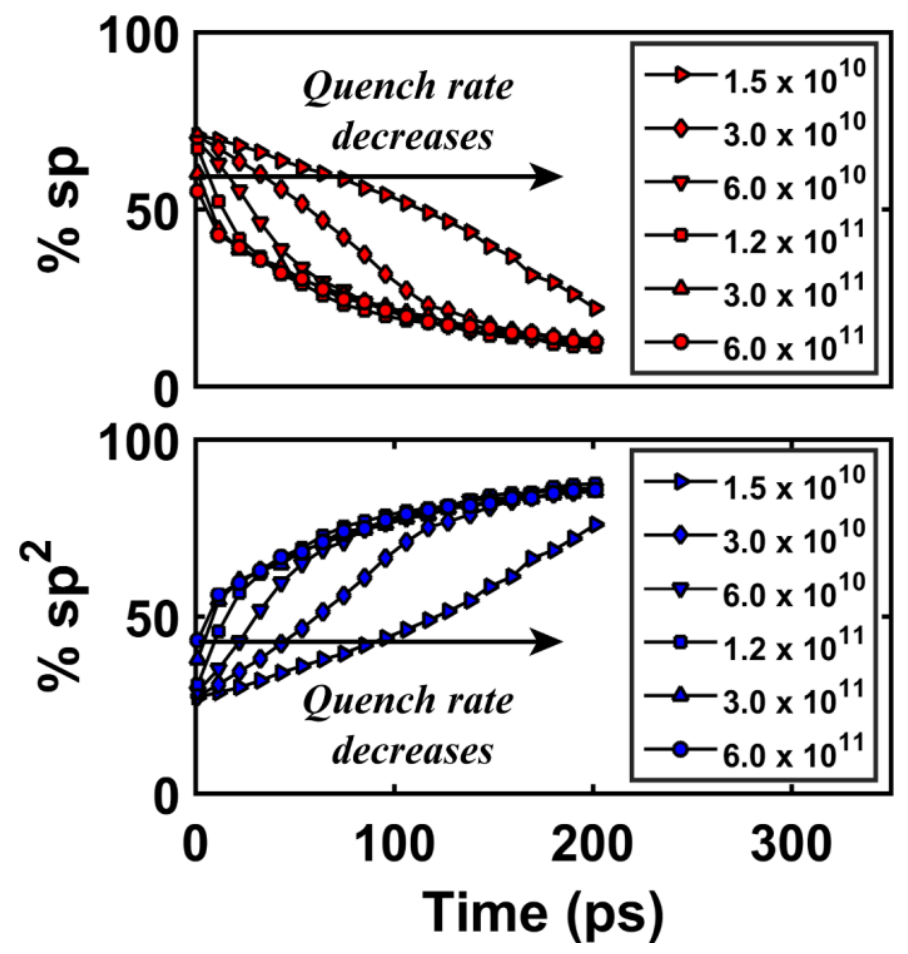

Figure 11: Variation of carbon hybridization during exponential quenching for different quench rates 


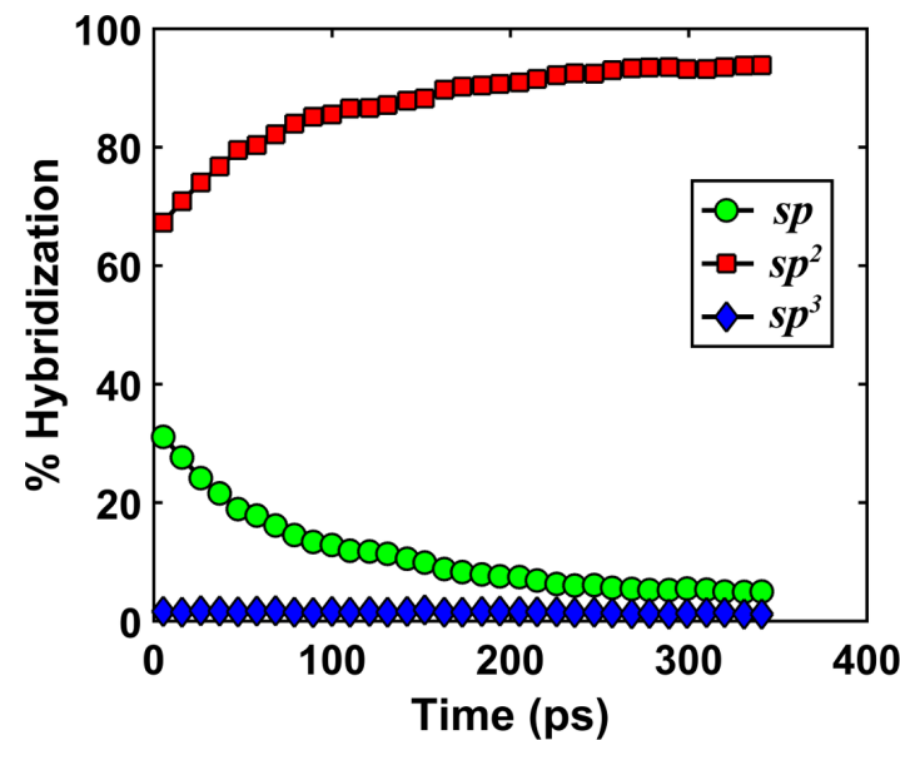

Figure 12: Variation of carbon hybridization during a long annealing stage at 3,000 $\mathrm{K}$ for amorphous carbon at density $1.0 \mathrm{~g} / \mathrm{cc}$. The starting stage is a quenched structure (from 10,000 K to $3,000 \mathrm{~K}$ ) using a linear quench rate, $Q=33.3 \mathrm{~K} / \mathrm{ps}$. Data are averaged over two independent samples. 

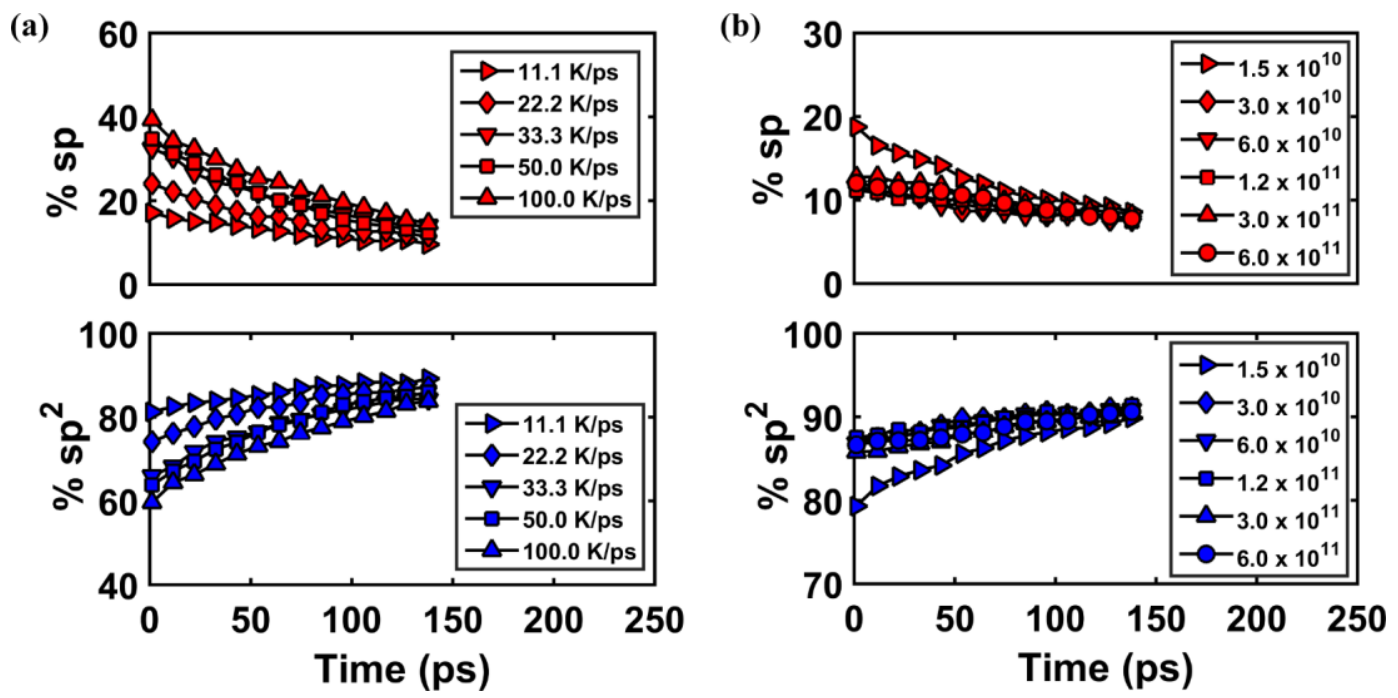

Figure 13: (a) Evolution of carbon hybridization during annealing at 3,000 K, for structures that were generated using various linear quench rates. (b) Carbon hybridization during annealing for various exponential quench rates. 

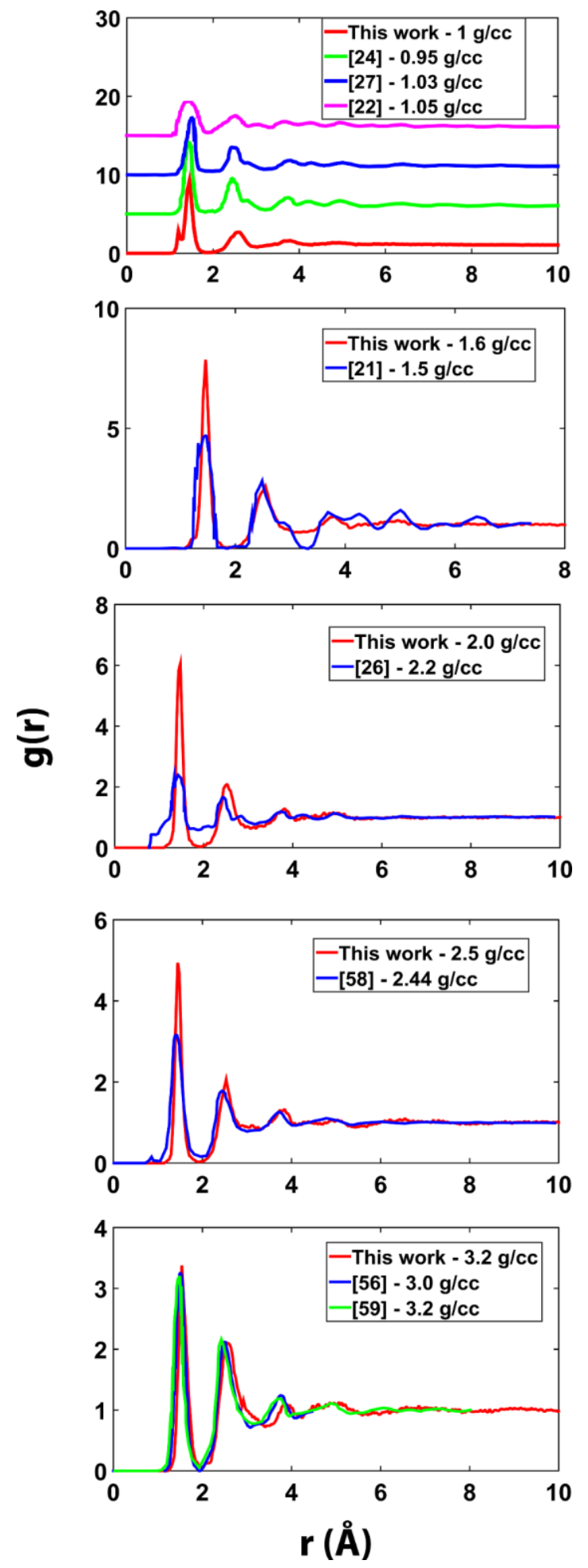

Figure 14: Comparison of pair correlation functions with models in literature, at various densities of $a-C$. The references for the experimental comparisons are indicated in legends. 


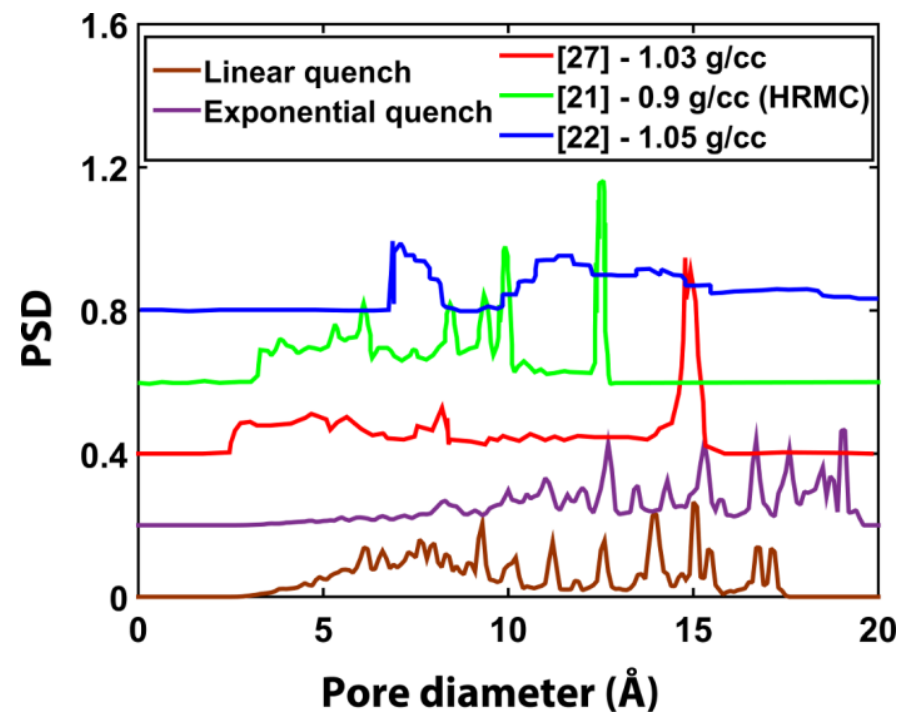

Figure 15: Comparison of PSD for $1 \mathrm{~g} / \mathrm{cc} a-C$ generated via linear and exponential quench routes, with other models at $\sim 1 \mathrm{~g} / \mathrm{cc}$ from literature. 


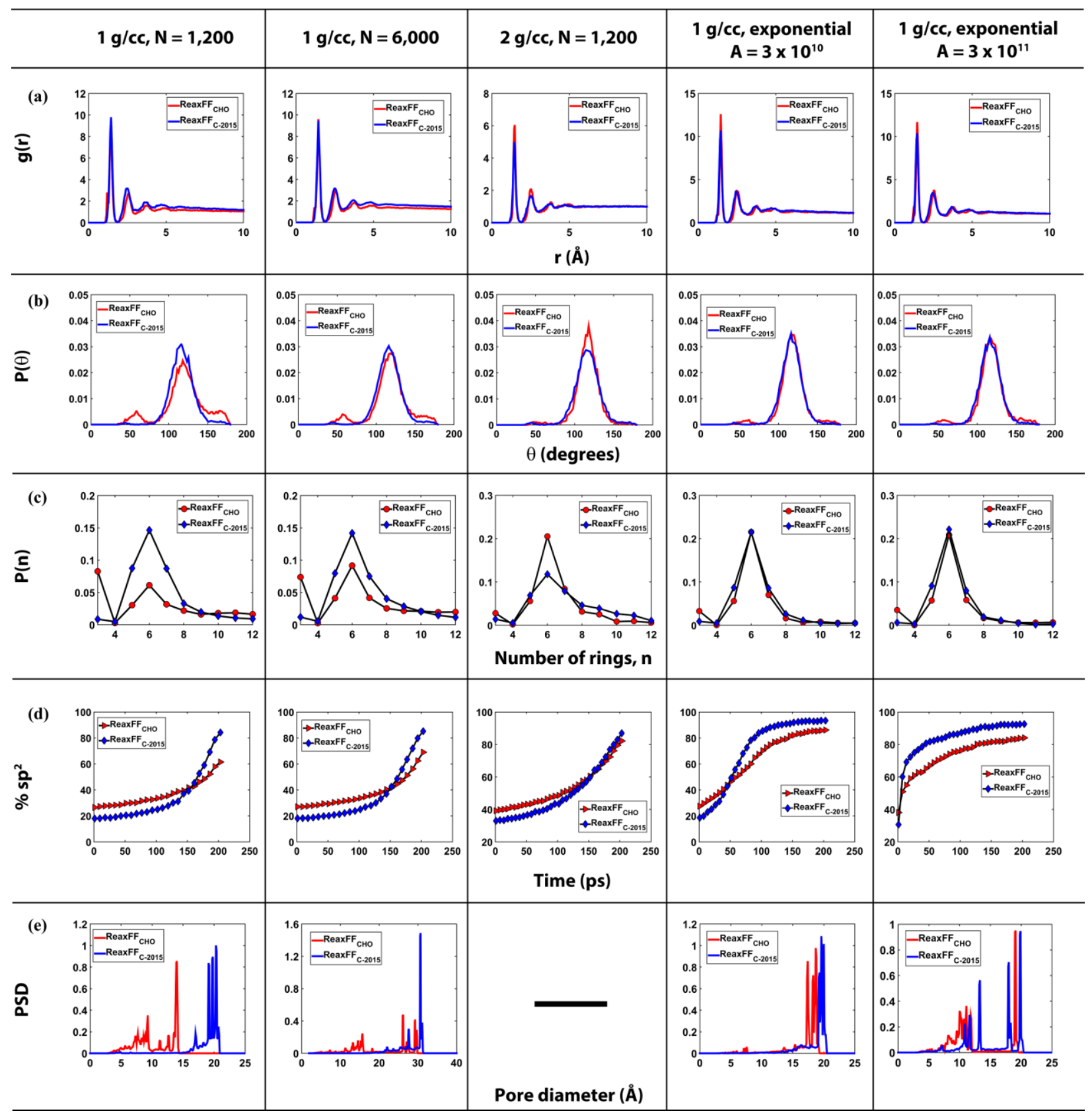

Figure 16: Comparison of structural features of $a-C$ between the $R e a x F F_{C H O}$ and a newer implementation of ReaxFF, ReaxFF $F_{C-2015}$ including (a) g(r), (b) angle distribution, (c) ring size distribution, (d) $s p^{2}$ hybridization and (e) pore size distribution, for various simulation parameters as described in Table 3. PSD for $2 \mathrm{~g} / \mathrm{cc}$ structure (panel (e)) is ill-defined (refer to text), and is omitted. 


\section{References}

[1] D.R. McKenzie, D. Muller, B.A. Pailthorpe, Compressive stress induced formation of thin-film tetrahedral amorphous carbon, Phys. Rev. Lett. 67 (1991) 773-776.

[2] H.K. Tran, C.E. Johnson, D.J. Rasky, F.C.L. Hui, M.-T. Hsu, Y.K. Chen, Phenolic Impregnated Carbon Ablators (PICA) for Discovery class missions, in: 31st AIAA Thermophys. Conf. New Orleans, 1996.

[3] A.S. V Pulickel, M.B. Chaudhari, Pyrolysis modelling and experimentation for thermophysical properties of char formed from ablative material, Int. J. Appl. Res. Mech. Eng. 1 (2011) $1-5$.

[4] Y. Liu, A. Erdemir, E.I. Meletis, A study of the wear mechanism of diamond-like carbon films 1, Surf. Coatings Technol. 82 (1996) 48-56.

[5] J. Robertson, Diamond-like amorphous carbon, Mater. Sci. Eng. R Reports. 37 (2002) $129-281$.

[6] H. Tsai, D.B. Bogy, Characterization of diamondlike carbon films and their application as overcoats on thin-film media for magnetic recording, J. Vac. Sci. Technol. A. 5 (1987) $3287-3312$.

[7] L. Colombo, A. Fasolino, Computer-Based Modeling of Novel Carbon Systems and Their Properties: Beyond Nanotubes, Carbon Materaials Chem. Phys. (2010).

[8] H.U. Jäger, K. Albe, Molecular-dynamics simulations of steady-state growth of iondeposited tetrahedral amorphous carbon films, J. Appl. Phys. 88 (2000) 1129. 
[9] M. Kaukonen, R.M. Nieminen, Atomic-scale modelling of the ion-beam-induced growth of amorphous carbon, Phys. Rev. B. 61 (2000) 2806-2811.

[10] D. Beeman, J. Silverman, R. Lynds, M.R. Anderson, Modeling studies of amorphous carbon, Phys. Rev. B. 30 (1984) 870-875.

[11] J. Lawson, D. Srivastava, Formation and structure of amorphous carbon char from polymer materials, Phys. Rev. B. 77 (2008) 144209.

[12] P.J.F. Harris, Fullerene-like models for microporous carbon, J. Mater. Sci. 48 (2012) 565577.

[13] J.C. Palmer, K.E. Gubbins, Atomistic models for disordered nanoporous carbons using reactive force fields, Microporous Mesoporous Mater. 154 (2012) 24-37.

[14] T.X. Nguyen, N. Cohaut, J.-S. Bae, S.K. Bhatia, New method for atomistic modeling of the microstructure of activated carbons using hybrid reverse Monte Carlo simulation., Langmuir. 24 (2008) 7912-7922.

[15] N. Quirke, J.P.R.B. Walton, Modelling the phase behavior of a fluid within a pore, Chem. Phys. Lett. 129 (1986) 382-386.

[16] C. Lastoskie, K.E. Gubbins, N. Quirke, Pore size heterogeneity and the carbon slit pore: A density functional theory model, Langmuir. 9 (1993) 2693-2702.

[17] Q. Wang, J.K. Johnson, Molecular simulation of hydrogen adsorption in single-walled carbon nanotubes and idealized carbon slit pores, J. Chem. Phys. 110 (1999) 577-586.

[18] S.K. Bhatia, A.L. Myers, Optimum Conditions for Adsorptive Storage Optimum Conditions for Adsorptive Storage, Langmuir. 22 (2006) 1688-1700.

[19] M.J. Biggs, a. Buts, Virtual porous carbons: what they are and what they can be used for, 
Mol. Simul. 32 (2006) 579-593.

[20] R. Powles, N. Marks, D. Lau, Self-assembly of $\mathrm{sp}^{2}$-bonded carbon nanostructures from amorphous precursors, Phys. Rev. B. 79 (2009) 75430.

[21] S.K. Jain, R.J.-M. Pellenq, J.P. Pikunic, K.E. Gubbins, Molecular modeling of porous carbons using the hybrid reverse Monte Carlo method, Langmuir. 22 (2006) 9942-9948.

[22] J.C. Palmer, J.K. Brennan, M.M. Hurley, a. Balboa, K.E. Gubbins, Detailed structural models for activated carbons from molecular simulation, Carbon 47 (2009) 2904-2913.

[23] G. Opletal, T. Petersen, B. O’Malley, I. Snook, D.G. McCulloch, N.A. Marks, et al., Hybrid approach for generating realistic amorphous carbon structure using metropolis and reverse Monte Carlo, Mol. Simul. 28 (2002) 927-938.

[24] A.H. Farmahini, S.K. Bhatia, Hybrid Reverse Monte Carlo simulation of amorphous carbon: Distinguishing between competing structures obtained using different modeling protocols, Carbon 83 (2015) 53-70.

[25] J. Pikunic, C. Clinard, N. Cohaut, K.E. Gubbins, J.-M. Guet, R.J.-M. Pellenq, et al., Structural Modeling of Porous Carbons: Constrained Reverse Monte Carlo Method, Langmuir. 19 (2003) 8565-8582.

[26] T. Petersen, I. Yarovsky, I. Snook, D.G. McCulloch, G. Opletal, Microstructure of an industrial char by diffraction techniques and Reverse Monte Carlo modelling, Carbon 42 (2004) 2457-2469.

[27] A.H. Farmahini, S.K. Bhatia, Effect of structural anisotropy and pore-network accessibility on fluid transport in nanoporous $\mathrm{Ti}_{3} \mathrm{SiC}_{2}$ carbide-derived carbon, Carbon 103 (2016) 16-27. 
[28] G. Galli, R.M. Martin, R. Car, M. Farrinello, Structural and Electronic Properties of Amorphous Carbon, Phys. Rev. Lett. 62 (1989) 555-558.

[29] C.Z. Wang, K.M. Ho, Structure, dynamics, and electronic properties of diamondlike amorphous carbon, Phys. Rev. Lett. 71 (1993) 1184-1187.

[30] N. Marks, Generalizing the environment-dependent interaction potential for carbon, Phys. Rev. B. 63 (2000) 35401.

[31] N. Marks, Modelling diamond-like carbon with the environment-dependent interaction potential, J. Physics. Condens. Matter. 14 (2002) 2901-2927.

[32] I. Suarez-Martinez, N. a. Marks, Effect of microstructure on the thermal conductivity of disordered carbon, Appl. Phys. Lett. 99 (2011) 33101.

[33] Y. Shi, A mimetic porous carbon model by quench molecular dynamics simulation., J. Chem. Phys. 128 (2008) 234707.

[34] J.C. Palmer, A. Llobet, S.H. Yeon, J.E. Fischer, Y. Shi, Y. Gogotsi, et al., Modeling the structural evolution of carbide-derived carbons using quenched molecular dynamics, Carbon 48 (2010) 1116-1123.

[35] M.A. Makeev, J.W. Lawson, D. Srivastava, Kinetics of formation and thermal and mechanical properties of char obtained by ultra-high temperature pyrolysis of polyethylene via molecular dynamics simulations, in: Mater. Charact. Comput. Model., 2009.

[36] M.A. Makeev, D. Srivastava, Thermal properties of char obtained by pyrolysis: A molecular dynamics simulation study, Appl. Phys. Lett. 95 (2009) 181908.

[37] A.C.T. van Duin, S. Dasgupta, F. Lorant, W. a. Goddard, ReaxFF: A Reactive Force 
Field for Hydrocarbons, J. Phys. Chem. A. 105 (2001) 9396-9409.

[38] R. Ranganathan, S. Rokkam, T. Desai, P. Keblinski, P. Cross, R. Burnes, Modeling hightemperature diffusion of gases in micro and mesoporous amorphous carbon, J. Chem. Phys. 143 (2015) 84701.

[39] S. Rokkam, R. Ranganathan, D.W. Brenner, P. Keblinski, "Methodology development of atomistically-informed chemical kinetics model for rubber composite materials," NAVAIR STTR Phase II (Base) Final Report - 30 July 2014 and NAVAIR STTR Phase II (Option) Final Report - 5 June 2016, Contract No. N68335-13-C-0119, 2014.

[40] L. Li, M. Xu, W. Song, A. Ovcharenko, G. Zhang, D. Jia, The effect of empirical potential functions on modeling of amorphous carbon using molecular dynamics method, Appl. Surf. Sci. 286 (2013) 287-297.

[41] J. Rosen, O. Warschkow, D.R. McKenzie, M.M.M. Bilek, Amorphous and crystalline phases in thermal quench simulations of alumina., J. Chem. Phys. 126 (2007) 204709.

[42] J. Yu, J. Lucas, T. Wall, G. Liu, C. Sheng, Modeling the development of char structure during the rapid heating of pulverized coal, Combust. Flame. 136 (2004) 519-532.

[43] G. Walberg, Analytical study of diffusion-controlled char oxidation and its effect on steady-state ablation of plastic materials, NASA Tech. Rep. (TR R-242). (1966).

[44] S. Bhattacharya, J. Kieffer, Fractal dimensions of silica gels generated using reactive molecular dynamics simulations., J. Chem. Phys. 122 (2005) 94715.

[45] J. Tersoff, Empirical interatomic potential for carbon, with applications to amorphous carbon, Phys. Rev. Lett. 61 (1988) 2879-2882.

[46] D.W. Brenner, Empirical potential for hydrocarbons for use in simulating the chemical 
vapor deposition of diamond films, Phys. Rev. B. 42 (1990) 9458-9471.

[47] D.W. Brenner, O. a Shenderova, J. a Harrison, S.J. Stuart, B. Ni, S.B. Sinnott, A secondgeneration reactive empirical bond order (REBO) potential energy expression for hydrocarbons, J. Phys. Condens. Matter. 14 (2002) 783-802.

[48] K. Chenoweth, A.C.T. van Duin, W. a Goddard, ReaxFF reactive force field for molecular dynamics simulations of hydrocarbon oxidation., J. Phys. Chem. A. 112 (2008) 10401053.

[49] K. Chenoweth, S. Cheung, A.C.T. van Duin, W. a Goddard, E.M. Kober, Simulations on the thermal decomposition of a poly(dimethylsiloxane) polymer using the ReaxFF reactive force field., J. Am. Chem. Soc. 127 (2005) 7192-202.

[50] K.D. Nielson, A.C.T. van Duin, J. Oxgaard, W.-Q. Deng, W.A. Goddard, Development of the ReaxFF reactive force field for describing transition metal catalyzed reactions, with application to the initial stages of the catalytic formation of carbon nanotubes., J. Phys. Chem. A. 109 (2005) 493-499.

[51] K.D. Nielson, A.C.T. van Duin, J. Oxgaard, W.-Q. Deng, W. a Goddard, Development of the ReaxFF reactive force field for describing transition metal catalyzed reactions, with application to the initial stages of the catalytic formation of carbon nanotubes., J. Phys. Chem. A. 109 (2005) 493-9.

[52] S. Plimpton, Fast Parallel Algorithms for Short-Range Molecular Dynamics, J. Comput. Phys. 117 (1995) 1-19.

[53] J.C. Palmer, a. Llobet, S.-H. Yeon, J.E. Fischer, Y. Shi, Y. Gogotsi, et al., Modeling the structural evolution of carbide-derived carbons using quenched molecular dynamics, 
Carbon 48 (2010) 1116-1123.

[54] S.J. Plimpton, A.P. Thompson, Computational aspects of many-body potentials, MRS Bull. 37 (2012) 513-521.

[55] S. Le Roux, V. Petkov, ISAACS - interactive structure analysis of amorphous and crystalline systems, J. Appl. Crystallogr. 43 (2010) 181-185.

[56] L. Guttman, Ring structure of the crystalline and amorphous forms of silicon dioxide.pdf, J. Non. Cryst. Solids. 116 (1990) 145-147.

[57] T.F. Willems, C.H. Rycroft, M. Kazi, J.C. Meza, M. Haranczyk, Algorithms and tools for high-throughput geometry-based analysis of crystalline porous materials, Microporous Mesoporous Mater. 149 (2012) 134-141.

[58] G. Opletal, T. Petersen, B. O’Malley, I. Snook, D.G. McCulloch, N.A. Marks, et al., Hybrid approach for generating realistic amorphous carbon structure using metropolis and reverse Monte Carlo, Mol. Simul. 28 (2002) 927-938.

[59] T. Petersen, I. Yarovsky, I. Snook, D.G. McCulloch, G. Opletal, Microstructure of an industrial char by diffraction techniques and Reverse Monte Carlo modelling, Carbon 42 (2004) 2457-2469.

[60] F. Li, J. Lannin, Radial Distribution Function of Amorphous Carbon, Phys. Rev. Lett. 65 (1990) 1905-1908.

[61] K.W.R. Gilkes, P.H. Gaskell, J. Robertson, Comparison of neutron-scattering data for tetrahedral amorphous carbon with structural models, Phys. Rev. B. 51 (1995) 1230312312.

[62] S.G. Srinivasan, A.C.T. van Duin, P. Ganesh, Development of a ReaxFF Potential for 
Carbon Condensed Phases and Its Application to the Thermal Fragmentation of a Large Fullerene, J. Phys. Chem. A. 119 (2015) 571-580.

[63] J. Towns, T. Cockerill, M. Dahan, I. Foster, K. Gaither, A. Grimshaw, et al., Accelerating Scientific Discovery, Comput. Sci. Eng. 16 (2014) 62-74. 


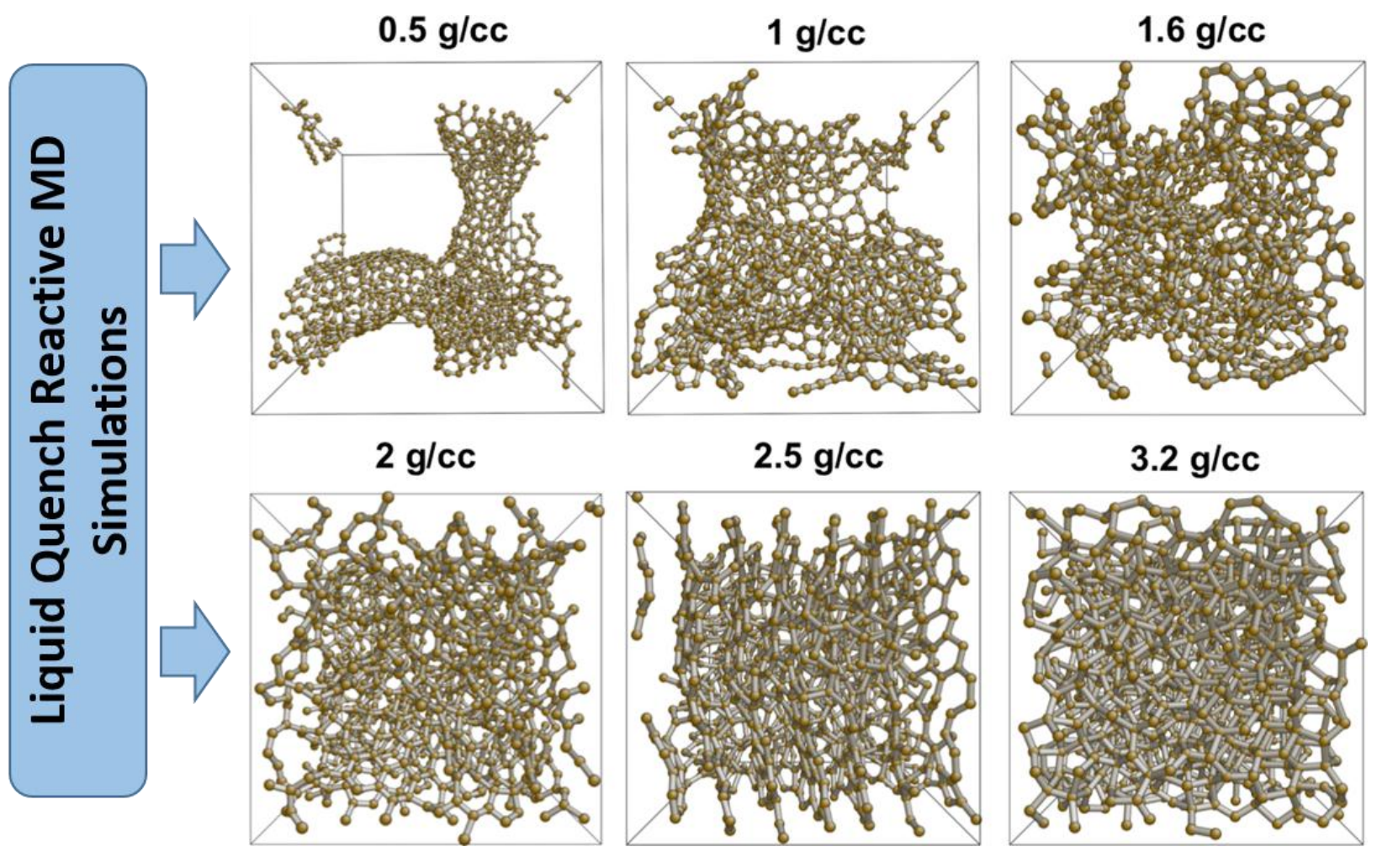

